\title{
La influencia de las alteraciones estructurales en los daños del terremoto de Amatrice, Italia (2016)
}

\section{The influence of structural alterations on the damages of the Amatrice earthquake, Italy (2016)}

\author{
$\underline{\text { José-Carlos Salcedo }}^{(*)}$, Manuel Fortea ${ }^{(*)}$
}

\section{RESUMEN}

El 24 de agosto de 2016, en los Apeninos italianos, se produjo un terremoto de magnitud 6,2 Mw que afectó principalmente a los núcleos de población de Amatrice, Accumoli, Arquata del Tronto y Pescara del Tronto, causando casi 300 muertos. En este artículo se presentan los resultados del trabajo de campo realizado al mes del evento sísmico sobre los restos constructivos del terremoto en el centro histórico de Amatrice. Se ha constatado que las estructuras originales de los edificios de este centro histórico patrimonial habían sido intervenidas (obras de ampliación, reforma, cambios de uso...) utilizando materiales y sistemas nuevos, como hormigón y acero, generándose sistemas estructurales mixtos anómalos, que no están adecuadamente considerados en el estado del conocimiento. Los resultados del estudio realizado demuestran la mayor vulnerabilidad sísmica de estas construcciones.

Palabras clave: Amatrice, terremoto, estructura de fábrica, patrimonio.

\section{ABSTRACT}

On August 24, 2016, in the Italian Apennines, an earthquake of magnitude 6.2 Mw occurred mainly affecting the villages of Amatrice, Accumoli, Arquata del Tronto and Pescara del Tronto, causing almost 300 deaths. This paper presents the results of a field work carried out one month after the seismic event on the remains of the earthquake in the historical city center of Amatrice. It has been verified that the original structures of the buildings of this historical heritage center had been manipulated (expansion works, refurbishments, changes in use...) using new materials and systems, such as concrete and steel, generating anomalous mixed structural systems, that are not considered in the state of knowledge. The results of the study carried out demostrate the higher seismic vulnerability of these constructions.

Keywords: Amatrice, earthquake, masonry structure, heritage.

(*) Arquitecto Dr. Universidad de Extremadura, Escuela Politécnica, Cáceres (España).

Persona de contacto/Corresponding author: jcsalcedo@unex.es (J.C. Salcedo).

ORCID: http://orcid.org/oooo-0002-7185-0286 (J.C. Salcedo); http://orcid.org/oooo-00o2-9700-9823 (M. Fortea).

Cómo citar este artículo/Citation: Salcedo, José-Carlos; Fortea, Manuel (2020). La influencia de las alteraciones estructurales en los daños del terremoto de Amatrice, Italia (2016). Informes de la Construcción, 72(559): e349. https://doi.org/10.3989/ic.71378.

Copyright: (C) 2020 CSIC. Este es un artículo de acceso abierto distribuido bajo los términos de la licencia de uso y distribución Creative Commons Reconocimiento 4.0 Internacional (CC BY 4.0). 


\section{INTRODUCCIÓN}

\subsection{Estado de la cuestión}

La ingeniería sísmica trata las estrategias estructurales a aplicar en los edificios de nueva construcción y con sistemas estructurales modernos, de hormigón y de acero fundamentalmente, pero no se ha ocupado en profundidad de las construcciones históricas. Las estructuras históricas, de fábricas (masonry structures) y de madera, de las que están hechas las construcciones de los centros históricos, son anteriores a la ingeniería sísmica y las estrategias de esta ingeniería no son directamente aplicables a ellas.

Las sociedades desarrolladas estábamos acostumbradas a ver arrasamientos de poblaciones por terremotos sólo en países subdesarrollados o en regiones con una muy alta peligrosidad sísmica. Pero además, en los últimos años, han tenido lugar en Europa una serie de episodios sísmicos de no mucha magnitud que han afectado a centros históricos completos, como L'Aquila o Amatrice (Italia), generando daños desproporcionados con respecto a la acción sísmica, a los que no estábamos acostumbrados. Daños de los que nos creíamos a salvo por cumplir "sobre el papel" las normas de construcción.

Ya se había constatado la alta vulnerabilidad sísmica del patrimonio, especialmente de las iglesias (1). El investigador S. Lagomarsino aportó importantes conclusiones a partir del estudio de daños del terremoto de L'Aquila (2009), ocurrido en la misma zona y con similar aceleración sísmica, analizando los mecanismos de colapso de 700 iglesias por el método de los macroelementos, que desarrolló a partir de sus experiencias previas en el terremoto de Umbría y las Marcas (1997).

Más recientemente, Del Gaudio (2) analiza la vulnerabilidad sísmica del patrimonio, pero no tiene en cuenta el grado de alteración sobre la estructura original.

Para esta investigación se parte de trabajos previos (3), en los que ya se ha acreditado, en el caso español del terremoto de Lorca (2011), que los edificios que registraron más daños estructurales, no fueron ni los nuevos ni los históricos, sino aquellos de sistemas estructurales mixtos: edificios históricos que habían sido intervenidos alterando sus estructuras con materiales y sistemas nuevos, fundamentalmente con hormigón armado.

Estos casos antecedentes, que afectaron sobre todo a los centros históricos (originariamente dotados de estructuras de fábricas y de madera, típicas del Sur de Europa) han puesto de manifiesto un inesperado parte de daños estructurales por terremotos y consiguientemente un elevado número de víctimas personales. Las características constructivas del patrimonio histórico hacen que los daños no se vean reducidos al intervenir en ellos aplicando normas estructurales, que sí tienen buenos resultados en edificios de nueva construcción. Es necesario por tanto estudiar la cuestión para mejorar la seguridad de las personas y de los bienes patrimoniales.

Hipótesis: Se plantea como hipótesis que estas construcciones de los centros históricos patrimoniales habrían sido alteradas de forma determinante en su constitución original, con obras de ampliación, reforma, cambio de uso, etc, y que estas alteraciones habrían incidido en su vulnerabilidad sísmica ${ }^{1}$.

Se sabe que el comportamiento de los edificios ante el sismo (vulnerabilidad sísmica) depende de dos factores. Por un lado, de la acción sísmica incidente (básicamente la aceleración horizontal producida por el sismo) y por otro, del sistema estructural del edificio (4). Se constata que ante la misma acción, los distintos edificios que componen un centro histórico presentan un comportamiento estructural diferente, de lo que se deduce que es determinante el sistema estructural, que en muchos casos en un centro histórico es el resultado de sucesivas intervenciones realizadas a lo largo del tiempo sobre una construcción histórica original.

Apenas existen estudios basados en casos reales sobre la seguridad estructural sísmica del patrimonio histórico que se hayan centrado en las alteraciones de la estructura resistente original, por lo que procede realizar una revisión de los planteamientos a la luz de la realidad de los recientes terremotos, con el análisis de la especificidad de sus materiales y sistemas constructivos, tanto de la construcción histórica original, como de las técnicas de intervención que se hayan empleado en ellos en los últimos años. Basta una somera revisión de la norma sísmica europea (Eurocódigo-8) (5), o de la escala de Mercalli (MMI), para comprobar que la rica complejidad constructiva de materiales y sistemas tradicionales con sus consiguientes mixtificaciones producidas en los últimos años a raíz de las intervenciones con hormigón, no están adecuadamente recogidas en el estado del conocimiento, y que las estructuras mixtas obtenidas no se comportan estructuralmente como los tipos sí recogidos.

La escala macrosísmica europea ${ }^{2}$ EMS-98 distingue los edificios de hormigón armado y los edificios de fábrica. Pero esta clasificación excesivamente simplista, no considera los casos puestos de manifiesto en esta investigación, por ejemplo en "fábricas" no contempla las bóvedas (de comportamiento estructural muy distinto al resto de fábricas), sólo los muros. Tampoco contempla los casos de estructuras mixtas originalmente de fábrica y recrecidas o reforzadas con hormigón, que alteran significativamente masa y rigidez.

\subsection{El terremoto de Amatrice (Italia, 2016)}

Entre agosto y octubre de 2016, en la región de Italia Central se registraron los episodios sísmicos destacables que se indican en la tabla 1.

De todos ellos, va a ser objeto de estudio el terremoto ocurrido el 24 de agosto de 2016, a las 01:36:32 (UTC) denominado

\footnotetext{
El peligro sísmico es la magnitud que cuantifica el riesgo debido a la zona geográfica sísmica en la que se encuentra el edificio. La vulnerabilidad sísmica cuantifica el riesgo debido únicamente al sistema estructural del edificio. Estos dos conceptos (peligro sísmico del emplazamiento y vulnerabilidad sísmica de la construcción), junto al daño sísmico potencial, determinan el riesgo sísmico.

${ }^{2}$ EMS-98 indica el grado en que un terremoto afecta a un lugar específico y contempla 12 grados. A diferencia de las escalas sísmicas de magnitud, que expresan la energía sísmica liberada por un terremoto.
} 
"de Amatrice" o “de Italia Central”. Véase mapa de situación en la figura 1. Fue el más destructivo pues aunque su mag$\operatorname{nitud}^{3}(6,2 \mathrm{Mw})$ fue menor que la del 30 de octubre de 2016 $(6,6 \mathrm{Mw})$, su hipocentro estuvo situado a menor profundidad $(4,4 \mathrm{~km}$ frente a 10,0 km) y más cerca de núcleos de población, por lo que produjo mayor aceleración y consiguientemente mayores daños.

El terremoto de Amatrice afectó a los núcleos de población (y a sus correspondientes centros históricos) de Amatrice (población de 2.650 habitantes ${ }^{4}$ en la que se produjeron ${ }^{5} 221$ muertos), Accumoli (667 habitantes y 11 muertos), Arquata del Tronto (1.322 habitantes) y Pescara del Tronto (135 ha- bitantes). Entre Arquata del Tronto y Pescara del Tronto se registraron 49 muertos.

En la misma zona geográfica, en el año 2009, se había producido el terremoto de L'Aquila (1), que guarda muchas similitudes con el de Amatrice en cuanto a la acción sísmica y daños producidos (véase tabla 2). Entre ambos epicentros existe una distancia de $44 \mathrm{Km}$ en línea recta. Véanse figuras 2 y 3 . Véase en la tabla 3 un resumen de datos geográficos de los principales núcleos de población afectados citados.

Los Apeninos Centrales son una de las áreas de mayor actividad sísmica en Italia. La cadena montañosa de los Apeninos se

Tabla 1. Episodios sísmicos estudiados.

\begin{tabular}{|c|c|c|c|c|c|c|c|}
\hline \multicolumn{2}{|c|}{ SERIE DE TERREMOTOS } & \multirow{2}{*}{ MAGNITUD } & \multicolumn{2}{|c|}{ COORDENADAS } & \multirow{2}{*}{$\frac{\text { PROF. }}{Z}$} & \multirow{2}{*}{$\begin{array}{l}\text { DISTANCIA A NÚCLEO } \\
\text { PRINICIPAL }\end{array}$} & \multirow{2}{*}{ NÚCLEOS DE POBLACIÓN CON MAYOR INTENSIDAD } \\
\hline FECHA & HORA & & $\bar{Y}$ & $\bar{x}$ & & & \\
\hline $24 / 08 / 2016$ & 01:36:32 (UTC) & $6,2 \mathrm{M}$ & $42,723^{\circ} \mathrm{N}$ & $13,188^{\circ} \mathrm{E}$ & $4,4 \mathrm{Km}$ & $10 \mathrm{Km}$ al SE Norcia & $\begin{array}{l}\text { IX: Amatrice, Accumoli. VIII: Arquata del Tronto, Castelsantangelo sul } \\
\text { Nera, Norcia, Montefortino, Visso, Preci, Montemonaco, Fluminata, } \\
\text { Campotosto, Comunanza, Balzo, Amandola, Cittareale. }\end{array}$ \\
\hline $24 / 08 / 2016$ & 02:33:29 (UTC) & $5,6 \mathrm{M}$ & $42,841^{\circ} \mathrm{N}$ & $13,153^{\circ} \mathrm{E}$ & $3,3 \mathrm{Km}$ & $7 \mathrm{Km}$ al SE Norcia & $\begin{array}{l}\text { VII: Norcia, Castelsantangelo sul Nera, Arquata del Tronto, } \\
\text { Amandola, Preci }\end{array}$ \\
\hline $26 / 10 / 2016$ & 17:10:37 (UTC) & $5,5 \mathrm{M}$ & $42,857^{\circ} \mathrm{N}$ & $13,023^{\circ} \mathrm{E}$ & $10,0 \mathrm{Km}$ & $8 \mathrm{Km}$ al ESE de Sellano & VII: Preci, Norcia, Castelsantangelo sul Nera, Fluminata \\
\hline $26 / 10 / 2016$ & 19:18:08 (UTC) & $6,1 \mathrm{M}$ & $42,934^{\circ} \mathrm{N}$ & $13,043^{\circ} \mathrm{E}$ & $10,0 \mathrm{Km}$ & $3 \mathrm{Km}$ al $\mathrm{W}$ de Visso & $\begin{array}{l}\text { VII: Castelsantangelo sul Nera, Fluminata, Valle e Castello, Visso, } \\
\text { Montemonaco, Trebbio. }\end{array}$ \\
\hline $30 / 10 / 2016$ & 06:40:19 (UTC) & $6,6 \mathrm{M}$ & $42,855^{\circ} \mathrm{N}$ & $13,088^{\circ} \mathrm{E}$ & $10,0 \mathrm{Km}$ & $6 \mathrm{Km}$ al $\mathrm{N}$ de Norcia & $\begin{array}{l}\text { VIII: Preci, Norcia, Visso, Castelsantangelo sul Nera, Fluminata, Valle } \\
\text { e Castelo }\end{array}$ \\
\hline
\end{tabular}

(Elaboración propia, a partir de datos del USGS).

Grados en la escala Mercali modificada (MMI) Modified Mercali Intensity, con la que trabaja USGS, de 12 grados (I not felt, II-III weak, IV light, V moderate, VI strong, VII very strong, VIII severe, IX violent, X extreme-1, XI extreme-2, XII-extreme-3). Es distinta de la escala macrosísmica europea (EMS-98) aunque ambas tienen 12 grados.

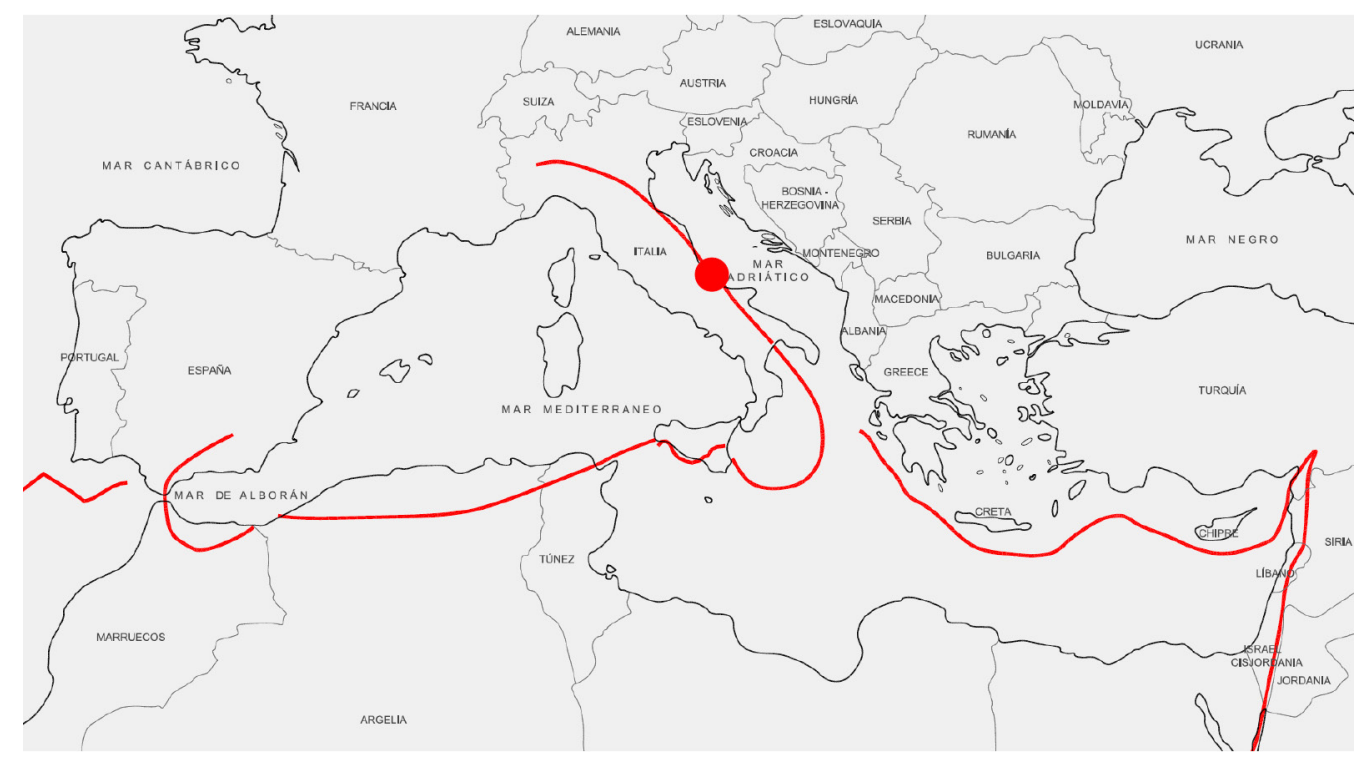

Figura 1. Situación del terremoto de Amatrice (6,2 Mw, 2016-08-24). Sobre el mapa de placas tectónicas. Fuente de Datos: USGS. Elaboración propia.

Tabla 2. Terremoto de L'Aquila (2009).

\begin{tabular}{|c|c|c|c|c|c|c|c|}
\hline \multicolumn{2}{|c|}{ SERIE DE TERREMOTOS } & \multirow{2}{*}{ MAGNITUD } & \multicolumn{2}{|c|}{ COORDENADAS } & PROF. & \multirow{2}{*}{$\begin{array}{c}\text { DISTANCIA A NÚCLEO } \\
\text { PRINICIPAL } \\
\end{array}$} & \multirow{2}{*}{ NÚCLEOS DE POBLACIÓN CON MAYOR INTENSIDAD } \\
\hline FECHA & HORA & & $\mathrm{Y}$ & $\mathrm{X}$ & Z & & \\
\hline $06 / 04 / 2009$ & 01:32:39 (UTC) & $6,3 \mathrm{M}$ & $42,334^{\circ} \mathrm{N}$ & $13,334^{\circ} \mathrm{E}$ & $8,8 \mathrm{Km}$ & $6 \mathrm{Km}$ al ESE de L'Aquila & $\begin{array}{l}\text { VIII: L’Aquila, Fossa, VII: Rocca di Cambio,Castel del Monte, Villa } \\
\text { Santa Lucia degli Abruzzi. }\end{array}$ \\
\hline
\end{tabular}

(Elaboración propia, a partir de datos del USGS).

\footnotetext{
3 «Earthquake Hazard Program: M6.2 - 10km SE of Norcia, Italy». earthquake.usgs.gov. USGS. 24 de agosto de 2016.

4 Amatrice alcanzó su pico poblacional en 1911, con 10.347 habitantes. Fuente: ISTAT (Istituto Nazionale di Statistica), Italia.

5 Datos oficiales facilitados por Protección Civil el 27 de agosto de 2016.
} 


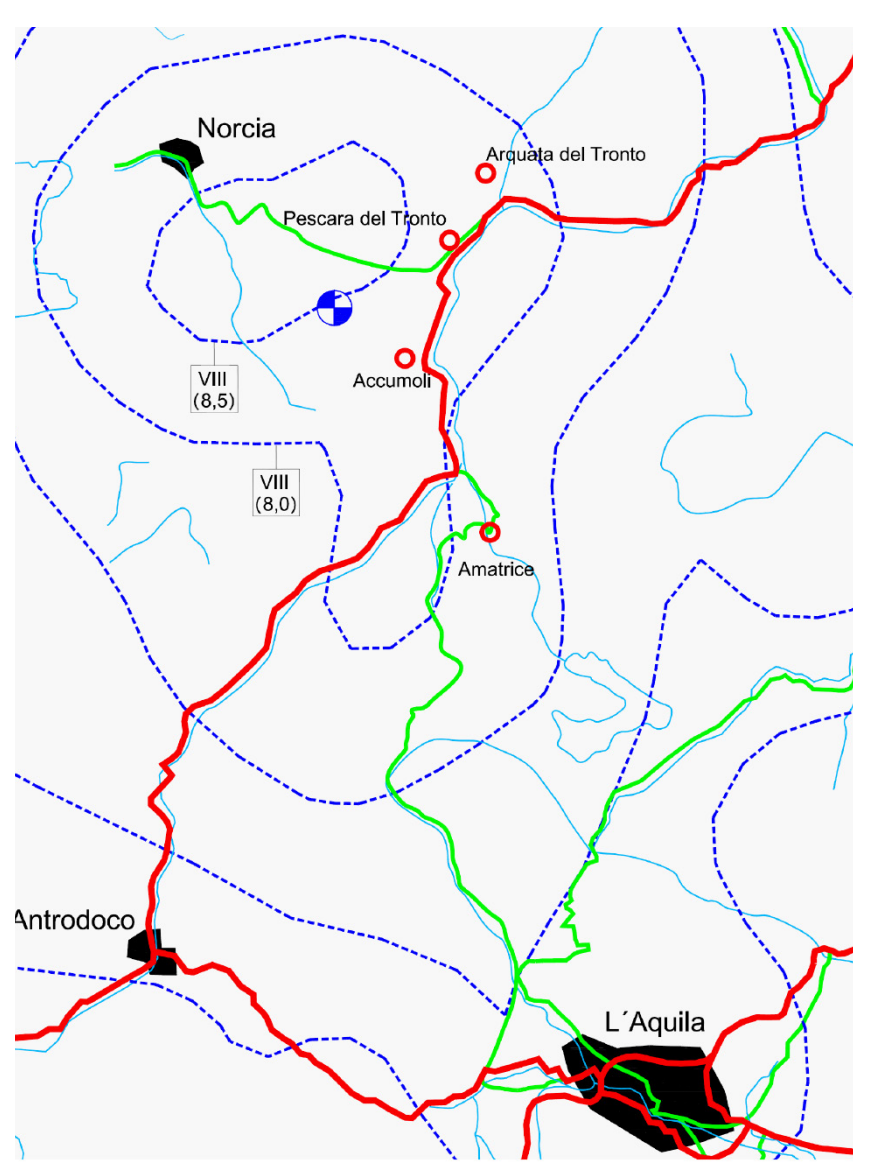

Figura 2. Área territorial afectada por el terremoto de Amatrice (6,2 Mw, 2016-08-24). Con epicentro a sólo 44 km al NNW de la localidad de L'Aquila, donde en 2009 tuvo lugar un sismo de magnitud 6,3 Mw. El mapa marca las líneas de intensidad sísmica. Los núcleos de población más afectados fueron Amatrice, Accumoli, Arquata del Tronto y Pescara del Tronto.

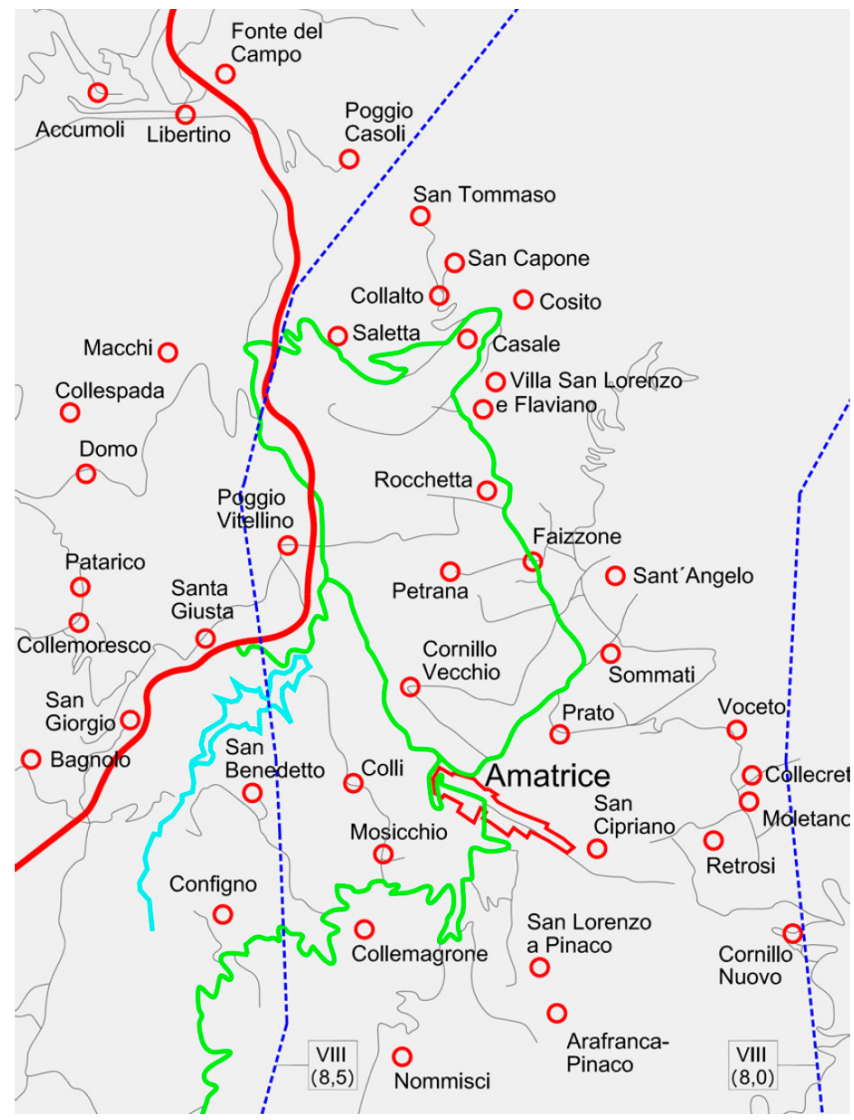

Figura 3. Afección a muchos núcleos de población. La particular ocupación del territorio de esta zona de Italia de los montes Apeninos, con gran número de núcleos de población y construcción en diseminado dispersa en el territorio, favorece la mayor probabilidad de existencia de pequeñas poblaciones muy próximas al epicentro sísmico. En el mapa se observan los núcleos de población en el entorno de Amatrice, afectados por intensidad VIII.

Tabla 3. Resumen de datos geográficos de los principales núcleos de población afectados por los eventos sísmicos de 2009 (L'Aquila) y 2016 (Amatrice).

\begin{tabular}{|c|c|c|c|c|c|c|c|}
\hline \multirow{2}{*}{ TERREMOTO } & \multirow{2}{*}{$\begin{array}{l}\text { NÚCLEO DE } \\
\text { POBLACIÓN }\end{array}$} & \multicolumn{2}{|c|}{ COORDENADAS } & \multirow{2}{*}{ Altitud } & \multirow{2}{*}{ Habitantes } & \multirow{2}{*}{$\begin{array}{l}\text { Víctimas } \\
\text { mortales }\end{array}$} & \multirow{2}{*}{$\begin{array}{c}\% \\
\text { Víctimas }\end{array}$} \\
\hline & & Latitud & Longitud & & & & \\
\hline L'AQUILA & L'Aquila & $42,349^{\circ} \mathrm{N}$ & $13,398^{\circ} \mathrm{E}$ & 726 & 73.150 & 308 & $0,42 \%$ \\
\hline \multirow{5}{*}{$\begin{array}{l}\text { AMATRICE } \\
24 / 08 / 2016\end{array}$} & Amatrice & $42,629^{\circ} \mathrm{N}$ & $13,289^{\circ} \mathrm{E}$ & 950 & 2.650 & 221 & $8,34 \%$ \\
\hline & Accumoli & $42,694^{\circ} \mathrm{N}$ & $13,246^{\circ} \mathrm{E}$ & 860 & 667 & 11 & $1,65 \%$ \\
\hline & Arquata del Tronto & $42,772^{\circ} \mathrm{N}$ & $13,295^{\circ} \mathrm{E}$ & 722 & 1.322 & \multirow{2}{*}{49} & \multirow{2}{*}{$3,36 \%$} \\
\hline & Pescara del Tronto & $42,751^{\circ} \mathrm{N}$ & $13,271^{\circ} \mathrm{E}$ & 715 & 135 & & \\
\hline & \multicolumn{4}{|c|}{ Media. } & 4.774 & 281 & $5,89 \%$ \\
\hline
\end{tabular}

formó del Mioceno al Plioceno, como resultado de la subducción de la placa Adriática debajo de la placa Euroasiática (6).

El hipocentro se produjo en las coordenadas $42,723^{\circ} \mathrm{N}$, $13,188^{\circ} \mathrm{E}$, a $4,4 \mathrm{~km}$ de profundidad, atribuyéndosele al terremoto principal (24 de agosto a las 3:36 h) una potencia de magnitud $^{6} \mathrm{ML}=\mathrm{MW}$ de 6,2. El epicentro se situó a $10 \mathrm{Km}$ al SE de Norcia. Véanse figuras 2 y 3.

La aceleración sísmica ha sido la esperada en los mapas de peligrosidad sísmica de referencia (7). La novedad ha sido el pésimo comportamiento estructural de determina- das construcciones (8) como las que son objeto de estudio, que ha sido superior en daños a lo descrito en la escala macrosísmica EMS-98 (9) con respecto a la aceleración producida.

La escasa profundidad del hipocentro es determinante de los daños (véase figura 4). Forma parte del estado del conocimiento (10) que un sismo de menor magnitud, pero cuyo hipocentro es muy superficial, produce una gran aceleración sísmica. Y la aceleración sísmica es la verdadera acción a las estructuras de los edificios, de la misma forma que es la aceleración de la gravedad la que origina el peso.

\footnotetext{
${ }^{6}$ Fuente: USGS, antes citada.
} 


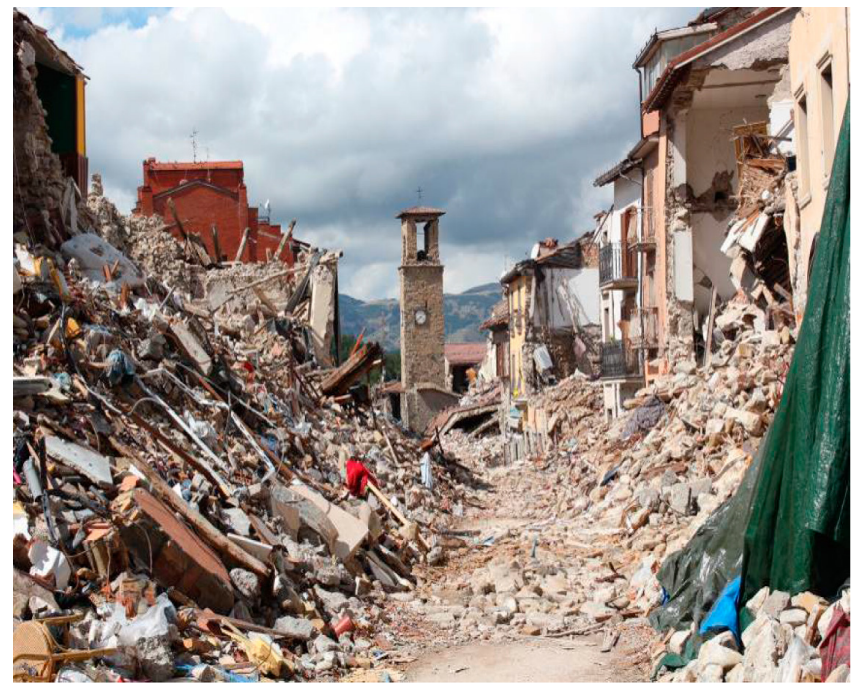

Figura 4. Calle principal del centro histórico de Amatrice (Corso Umberto I), con el caserío arrasado por el terremoto. Permanece en pie la torre Cívica, que en principio debería haber sido una de las construcciones más afectadas por su forma estructural y sin embargo, como el resto de las torres, fue uno de los pocos edificios no colapsados.

\section{METODOLOGÍA}

El método de investigación ha consistido en un trabajo de campo para evaluar los daños en los edificios de Amatrice después del terremoto.

En una primera fase se ha producido el traslado al núcleo de Amatrice de los investigadores, para la toma de datos in situ de los daños causados por el terremoto a las construcciones del centro 7 histórico, dotados de planos parcelarios y fotografías aéreas del estado previo al evento sísmico. Este trabajo de campo se realizó al mes de ocurrido el terremoto, fecha elegida porque los servicios de emergencia ya habían finalizado los trabajos de rescate y los restos constructivos aún no habían sido desescombrados, es decir, en el momento idóneo para el registro de los daños constructivos.

La obtención de los permisos de acceso fue compleja. Se contó con acreditaciones de la embajada española en Italia y del Rector de la Universidad de Extremadura.

En una segunda fase, de trabajo de gabinete, se ha procedido al análisis de toda la información recabada, para llegar a los resultados que se exponen en este trabajo.

Se definió un ámbito de estudio, en torno a la calle principal (Corso Umberto I), dentro de la zona roja ("zona rossa") que había sido delimitada por los servicios de rescate en el núcleo urbano de Amatrice. La zona roja coincidía con el centro histórico de la población.

Para la evaluación de los daños ha sido necesaria una clasificación que supere la simplista distinción de la EMS-98 entre edificios de fábrica y edificios de hormigón armado. A partir de experiencias previas de los autores en la evaluación de los daños del terremoto de Lorca (3) se han manejado las siguientes clasificaciones en la toma de datos, precisadas luego con el contraste de toda la información en trabajo de gabinete:

- Construcciones desde el punto de vista de su tipo estructural, simplificando tipos estructurales (o esquemas, o modelos) clarificados por primera vez por E. Torroja (11) y desarrolladas luego por otros autores como J.L de Miguel (12).

- Construcciones de pisos.

- Construcciones diáfanas (tipo iglesia).

- Construcciones esbeltas (tipo torre).

- Estructuras según la época de construcción, para aportar datos sobre su construcción original.

- Estructuras históricas.

- Estructuras recientes.

- Estructuras recientes sismo-resistentes.

- Edificaciones desde el punto de vista de su singularidad arquitectónica, porque existe una diferenciación en cuanto al detalle de los materiales y sistemas estructurales, y a la escala del edificio:

- Singulares

- Populares y racionalistas.

En cualquier caso y debido a la complejidad de las estructuras mixtificadas, fue necesario en la toma de datos un apartado de observaciones detalladas, para completar la descripción de cada una de las clasificaciones. Para resumir finalmente:

A) La siguiente tipificación de alteraciones en las estructuras desde el punto de vista de su vulnerabilidad sísmica (tabla 4):

Tabla 4. Tipificación de alteraciones en las estructuras.

\begin{tabular}{|l|l|}
\hline Tipo 1 & $\begin{array}{l}\text { Estructura histórica no alterada. } \\
\text { O con leves modificaciones que no alteran significativa- } \\
\text { mente su configuración estructural inicial. }\end{array}$ \\
\hline Tipo 2 & $\begin{array}{l}\text { Estructura histórica alterada. } \\
\text { Resultado de la alteración de su estructura histórica } \\
\text { original con nuevos sistemas estructurales recientes } \\
\text { (normalmente para ampliaciones, reformas o cambios } \\
\text { de uso. Distinguiendo: 2.1. Poco alterada. 2.2. Bastante } \\
\text { alterada. 2.3. Muy alterada. }\end{array}$ \\
\hline Tipo 3 & $\begin{array}{l}\text { Estructura reciente normal. } \\
\text { Edificios de estructura reciente completa sin diseño } \\
\text { sismo-resistente. }\end{array}$ \\
\hline Tipo 4 & $\begin{array}{l}\text { Estructura reciente sismo-resistente. } \\
\text { Edificios de estructura reciente completa con diseño } \\
\text { sismo-resistente. }\end{array}$ \\
\hline
\end{tabular}

B) Y los siguientes grados de daños estructurales (tabla 5).

Tabla 5. Grados de daños estructurales.

\begin{tabular}{|l|l|}
\hline Grado 1 & Colapso completo. \\
\hline Grado 2 & $\begin{array}{l}\text { Colapso parcial. } \\
\text { De cubierta, muros medianeros, grandes témpanos de } \\
\text { muros o de cubiertas. Pero deja huecos para las personas. }\end{array}$ \\
\hline Grado 3 & $\begin{array}{l}\text { Daños estructurales graves. } \\
\text { Sin colapso total o parcial. Mantiene el volumen edifi- } \\
\text { cado. }\end{array}$ \\
\hline Grado 4 & $\begin{array}{l}\text { Daños estructurales menores. } \\
\text { Presenta daños en la estructura resistente no pertene- } \\
\text { cientes a los grados anteriores. }\end{array}$ \\
\hline
\end{tabular}

\footnotetext{
El resto del núcleo de población, constituido por construcciones recientes, no registró tantos daños estructurales y no va a ser objeto de estudio.
} 


\section{RESULTADOS}

De la aplicación de la metodología descrita a los datos recabados en el trabajo de campo al mes del evento sísmico:

\subsection{Cómputo de edificios y sus características físicas}

El número de inmuebles estudiados (área de estudio dentro de la "zona rossa") ha sido de 74 unidades. La superficie total de las parcelas fue de $11.707 \mathrm{~m}^{2}$. Y la superficie construida del conjunto de los edificios fue de $31.857 \mathrm{~m}^{2}$, con un volumen edificado: $81.697 \mathrm{~m}^{3}$.

\subsection{Tipos de edificios según su tipología estructural}

- Estructuras históricas singulares diáfanas (tipo iglesia): 4, que han representado el 5,41\% de los inmuebles estudiados. Se trató de las parcelas S106 (centro cultural Consiglio Comunale, antigua iglesia de S. Giuseppe, s. XVII), S208 (iglesia del Purgatorio, s. XVIII), N400 (iglesia de S. Giovanni, s. XVI) y N501 (iglesia de S. Agostino, s. XV).

- Estructuras históricas singulares esbeltas (tipo torre): 2, que han representado el 2,70\%. Parcelas N301 (torre Cívica, s. XIII) y N5O3 (torre-campanario de la iglesia de S. Agostino, s. XV).

- Estructuras históricas singulares de pisos (palacetes): 2, que han representado el 2,70\%. Parcelas N2O2 y S412.

- El total de estructuras históricas singulares (8 unidades) ha representado el 10,81\% del número de parcelas de la zona de estudio.

- Edificios construidos de nueva planta con estructura reciente de pisos, de antigüedad menor de 76 años (completa de forjados de hormigón armado y pilares desde cimentación): 4 unidades, que han representado el 5,41\% del número de parcelas de la zona de estudio. Parcelas $\mathrm{S}_{301}, \mathrm{~S} 403$ (edificio de seis plantas de ladrillo visto conocido como el "edificio rojo"), S416 y S501.

- Edificios de estructura reciente sismo-resistente: No consta que existiera alguno.

- Resto de construcciones con estructura histórica, de antigüedad mayor de 67 años (aunque puedan haber registrado recientes reformas y/o ampliaciones y/o cambios de uso): 62 unidades, que representan el 83,78\%.

\subsection{Tabla resumen de resultados}

Véase en la tabla 6 el resumen de los resultados.

\subsection{Plano resumen de resultados}

El plano resumen (figura 5) describe gráficamente la zona de estudio en tres dibujos:

- En el primero se ha indicado el tipo de estructura, distinguiendo las estructuras históricas singulares, el resto de estructuras históricas (populares y racionalistas) y las estructuras recientes (completas de nueva planta). A su vez, dentro de las primeras, se destacan las estructuras esbeltas (tipo torre), las estructuras diáfanas (tipo iglesia) y las estructuras de pisos (tipo palacete).

- En el segundo se han indicado los daños estructurales registrados en el terremoto del 24 de agosto de 2016, distinguiendo los edificios que han tenido colapso total, colapso parcial y otros daños estructurales (sin colapsos).
- El tercero es un dibujo de síntesis en el que se han señalado las alteraciones sufridas sobre la estructura original, distinguiendo estructuras históricas no alteradas, estructuras históricas alteradas y estructuras recientes (todas son no alteradas). Dentro de las segundas se han establecido subcategorías para distinguir las poco, bastante y muy alteradas.

\subsection{Descripción de las alteraciones sobre las estructuras históricas originales que han sido identificadas en los restos}

Amatrice tiene un centro histórico de valor patrimonial. También está considerado uno de los pueblos " "piu bello d'Italia". Sin embargo, del análisis forense de los restos se constata claramente una intensa alteración de las estructuras históricas originales. La mayoría de los edificios habían visto alterada su estructura original con ampliaciones y/o reformas y/o cambios de uso (esta última alteración especialmente en las plantas bajas para los locales comerciales derivados de la actividad turística del centro histórico).

En estas obras no se han empleado las técnicas de las estructuras históricas (fábricas y entramados de madera anteriormente descritas) sino que se ha detectado el empleo sistemático de materiales y técnicas nuevas. Además, estas operaciones se llevan haciendo (por la fecha de las distintas soluciones constructivas y materiales encontrados en la inspección forense de los restos), desde hace muchos años, desde la segunda mitad del s. XX.

A continuación se tipifican las intervenciones más frecuentes que se han detectado y que por la extensión del artículo, sólo pueden ser relacionadas:

- Capas de mortero/hormigón y nuevos solados pesados añadidos sobre entramados de piso (horizontales) de madera preexistentes, que multiplican por enteros la masa inicial.

- Nuevos forjados construidos sobre entramados de piso (horizontales) de madera preexistentes, que multiplican por enteros la masa inicial y cuyas viguetas no se introducen a eje de los muros.

- Capas de mortero/hormigón sobre entramados de cubierta (inclinados) de madera, que multiplican por enteros la masa inicial.

- Capas de hormigón sobre bóvedas, que las cargan de manera anómala.

- Nuevos forjados en ampliaciones en altura, apoyados sobre muros de fábrica preexistentes, recrecidos con muros de fábricas de ladrillo recientes.

- Nueva estructura vertical de pilares (y sus forjados) en ampliaciones en altura, apoyados sobre muros de fábrica preexistentes.

- Nuevos faldones de cubierta con vigas de acero en ampliaciones en altura.

- Nuevos faldones de cubierta con forjados de hormigón en ampliaciones en altura.

- Apertura de grandes huecos en muros, para nuevas puertas y ventanas, escaparates, o para lograr diafanidad en planta.

- Refuerzos de acero a las fábricas de manera anómala.

- Refuerzos de hormigón en muros de manera anómala, con aumento significativo de masa.

\footnotetext{
${ }^{8}$ Está considerado uno de los parajes más hermosos de Italia, gracias a su patrimonio arquitectónico y urbano, y a su patrimonio natural. Se encuentra en el Parque Nacional del Gran Sasso y Montes de la Laga, creado en 1991, que tiene una superficie de 2.014 kilómetros cuadrados
} 
Tabla 6. Resumen de resultados.

\begin{tabular}{|c|c|c|c|c|c|c|c|c|c|c|c|c|c|c|c|c|}
\hline \multicolumn{3}{|c|}{ LOCALIZACIÓN } & \multicolumn{6}{|c|}{ MEDICIÓN } & \multicolumn{6}{|c|}{ DETALLE DE LA ESTRUCTURA } & \multicolumn{2}{|c|}{$\begin{array}{l}\text { TIPO DE ALTERACIÓN Y } \\
\text { GRADO DE DAÑOS }\end{array}$} \\
\hline REFEF & ENCIA & NÚMEROS & $\begin{array}{l}\text { Asignaci } \\
\text { sup.cons. }\end{array}$ & $\begin{array}{l}\text { nes para } \\
\text { volumen }\end{array}$ & & SUPERFICIE & Y VOLUMEN & & & & 莺 & 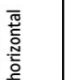 & 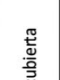 & 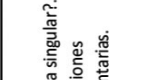 & IPO DE & DO DE \\
\hline MANZANA & PARCELA & PUERTAS & $\begin{array}{c}\text { Plantas } \\
\text { equiv. } \\
\text { sup.cons. }\end{array}$ & $\begin{array}{l}\text { Coef. de } \\
\text { vol. }\end{array}$ & $\begin{array}{l}\text { SUPERF. } \\
\text { PARCELA }\end{array}$ & $\begin{array}{l}\text { SUPERF. } \\
\text { CONST. }\end{array}$ & VOLUMEN & PLANTAS & 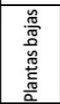 & 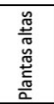 & $\begin{array}{l}\frac{0}{2} \\
\text { 尊 }\end{array}$ & $\begin{array}{l}\stackrel{\circ}{2} \\
\text { i }\end{array}$ & 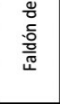 & 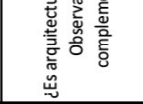 & \begin{tabular}{|c|} 
EN LA \\
ESTRUCTURA
\end{tabular} & $\begin{array}{c}\text { ESTRUCTURAL } \\
\text { ES }\end{array}$ \\
\hline & $\begin{array}{l}5101 \\
5102\end{array}$ & $\begin{array}{ll}150-148 \\
10-149\end{array}$ & 3 & 1 & 88,90 & $\begin{array}{ll}266,70 \\
6,57\end{array}$ & 666,75 & 3 & \begin{tabular}{|l|l|} 
MFP \\
MFC
\end{tabular} & $\begin{array}{l}\text { MFP } \\
\end{array}$ & \begin{tabular}{|l|l|}
$\mathrm{E}+\mathrm{H}$ \\
\end{tabular} & $\mathrm{E}+\mathrm{H}$ & $\mathrm{E}$ & NO & 2.1 & 1 \\
\hline & 5103 & \begin{tabular}{|l|}
$1462-144$ \\
$142-140$ \\
\end{tabular} & $\frac{3}{3}$ & $\frac{1}{1}$ & $\frac{64,19}{98,00}$ & $\frac{192,57}{294,00}$ & $\frac{481,43}{735,00}$ & $\frac{3}{3}$ & \begin{tabular}{|l|} 
MFP \\
\end{tabular} & MFP & \begin{tabular}{|l|l|}
$\mathrm{H}+\mathrm{H}$ \\
$\mathrm{H}$
\end{tabular} & \begin{tabular}{|l|l|}
$+H$ \\
$E+H$
\end{tabular} & \begin{tabular}{|l|l|}
$\mathrm{FHI}$ \\
$\mathrm{E}+\mathrm{H}$ \\
\end{tabular} & $\begin{array}{l}\text { NO } \\
\text { NO }\end{array}$ & $\frac{2.3}{2.1}$ & $\frac{1}{1}$ \\
\hline S1 & S104 & 138-136 & 3 & 1,2 & 78,31 & 234,93 & 704,79 & 3 altas & MFP & $\mathbf{P}$ & \begin{tabular}{|l|l|l}
$F H$ \\
\end{tabular} & $\mathrm{FH}$ & FHI & NO & 2.2 & 2 \\
\hline & S105 & 134-132 & 2 & 1,2 & 184,07 & 368,14 & $1.104,42$ & 2 altas & $\mathrm{MF}$ & MF & $E+H$ & $E+H$ & $E+H$ & NO & 2.1 & 3 \\
\hline & S106 & $130-128$ & 1,5 & 1 & 429,29 & 643,94 & $1.609,84$ & Equiv a 3 & MF & $\mathrm{MF}$ & $E+H$ & & $\mathrm{E}$ & $\begin{array}{l}\text { Histoŕrca singular } \\
\text { iglesia }\end{array}$ & 2.1 & 2 \\
\hline & 5201 & $126-124$ & 3 & 1,2 & 170,83 & 512,49 & $1.537,47$ & 3 altas & MFP & $\mathrm{MF}$ & $\mathrm{FH}$ & $\mathrm{FH}$ & $E+H$ & NO & 2.2 & 3 \\
\hline & S202 & $122-118$ & 2 & 1,2 & 109,64 & 219,28 & 657,84 & 2 altas & MFP & $\mathrm{MF}$ & $\mathrm{FH}$ & $\mathrm{FH}$ & $\mathrm{E}+\mathrm{H}$ & NO & 2.2 & 3 \\
\hline & $\begin{array}{l}\mathbf{S 2 0 3} \\
\mathbf{5 2 0 4}\end{array}$ & $\begin{array}{l}116-114 \\
1112-10\end{array}$ & $\frac{2}{3}$ & 1 & $\begin{array}{r}111,80 \\
57,75\end{array}$ & $\begin{array}{r}223,60 \\
173,25\end{array}$ & $\begin{array}{l}559,00 \\
433,13\end{array}$ & $\frac{2}{3}$ & \begin{tabular}{|l|} 
MFP \\
MFP
\end{tabular} & $\begin{array}{l}\text { MF } \\
M F\end{array}$ & \begin{tabular}{|l|l|}
$F H$ \\
$F H$ \\
$F H$
\end{tabular} & $\begin{array}{ll}F H \\
F H\end{array}$ & \begin{tabular}{|l|l}
$E+H$ \\
$E+H$
\end{tabular} & $\begin{array}{l}\text { NO } \\
\text { NO }\end{array}$ & 2.2 & 3 \\
\hline & 5204 & \begin{tabular}{|c|}
$112-110$ \\
$108-106$
\end{tabular} & $\frac{3}{3}$ & $\frac{1}{1}$ & 74,57 & 223,71 & $\begin{array}{l}4559,28 \\
559\end{array}$ & 3 & \begin{tabular}{|l|} 
MFP \\
\end{tabular} & $\mathrm{MF}$ & $\mathrm{FH}$ & $\mathrm{FH}$ & $\mathrm{E}+\mathrm{H}$ & NO & 2.2 & 3 \\
\hline S2 & 5206 & $\begin{array}{ll}104-100 \\
\end{array}$ & 3 & 1 & 182,69 & 548,07 & $1.370,18$ & 3 & \begin{tabular}{|l|l|} 
MFP \\
\end{tabular} & $\mathrm{MF}$ & $\mathrm{FH}$ & $\mathrm{FH}$ & \begin{tabular}{|l|l|}
$\mathrm{FHII}$ \\
\end{tabular} & NO & 2.3 & 1 \\
\hline & S207 & 98 & 1 & 1 & 37,50 & 37,50 & 93,75 & 1 & MFP & MFP & $\mathrm{E}+\mathrm{H}$ & $\mathrm{E}+\mathrm{H}$ & E & \begin{tabular}{|c} 
Histortica aningular \\
iglesia
\end{tabular} & 2.2 & 3 \\
\hline & $\mathbf{5 2 0 8}$ & $96-92$ & 3 & 1 & 233,09 & 699,27 & $1.748,18$ & Equiv a 3 & MFP & MFP & $\mathrm{E}+\mathrm{H}$ & & E & \begin{tabular}{|l|} 
Histórica asingular \\
iglesia
\end{tabular} & 2.1 & 3 \\
\hline & S209 & & 3 & 1 & 54,19 & 162,57 & 406,43 & 3 & MFP & MFP & $E+H$ & $E+H$ & $E$ & \begin{tabular}{|l|} 
Histórica singular \\
ielesia
\end{tabular} & 2.3 & 2 \\
\hline & 5301 & 90 & 3 & 1,2 & $211,5 /$ & 832,11 & $2.498,13$ & 3 altas & $\begin{array}{ll}p \\
\end{array}$ & $\mathrm{p}$ & \begin{tabular}{|l|l|}
$+H$ \\
\end{tabular} & $\mathrm{FH}$ & $\begin{array}{rll}\mathrm{HHI} \\
\end{array}$ & 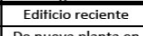 & 3 & 3 \\
\hline & S302 & $88-86$ & 3 & 1 & 175,93 & 527,79 & $1.319,48$ & 3 & MFP & MF & $\mathrm{FH}$ & $\mathrm{FH}$ & FHI & \begin{tabular}{|l|} 
De nueva planta en \\
este siglo
\end{tabular} & 2.2 & 3 \\
\hline 33 & S303 & $84-82$ & 3 & 1 & 236,82 & 710,46 & $1.776,15$ & 3 & MFP & $\mathrm{MF}$ & $\mathrm{FH}$ & $\mathrm{FH}$ & $\mathrm{E}+\mathrm{H}$ & \begin{tabular}{|l} 
De nueva planta en \\
este sigto
\end{tabular} & 2.1 & 3 \\
\hline & S304 & $80-72$ & 3 & 1,2 & 672,48 & $2.017,44$ & $6.052,32$ & 3 altas & MFP & $\mathrm{MF}$ & $\mathrm{FH}$ & $\mathrm{FH}$ & $E+H$ & $\begin{array}{l}\text { De nueva planta en } \\
\text { sete siglo }\end{array}$ & 2.1 & 2 \\
\hline & S401 & $70-68$ & 3 & 1 & 361,34 & $1.084,02$ & $2.710,05$ & 3 & MFP & MF & $\mathrm{FH}$ & $\mathrm{FH}$ & $E+1$ & 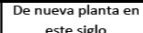 & 2.2 & 2 \\
\hline & 5402 & $66-64$ & 2 & 1 & 145,45 & 290,90 & 727,25 & 2 & \begin{tabular}{|l|l|} 
MFP \\
\end{tabular} & MF & \begin{tabular}{|l|l|}
$\mathrm{FH}$ \\
\end{tabular} & $\mathrm{FH}$ & \begin{tabular}{ll|}
$\mathrm{FHI}$ \\
\end{tabular} & & 2.2 & 1 \\
\hline & 5403 & $62-54$ & 5,5 & 1 & 180,39 & 992,15 & $2.480,36$ & 6 & $\begin{array}{ll}P \\
\end{array}$ & $\mathrm{P}$ & \begin{tabular}{|l|l|}
$\mathrm{FH}$ \\
\end{tabular} & $\mathrm{FH}$ & $\mathrm{FH}$ & Edificio reciente & 3 & 3 \\
\hline & 5404 & $52-46$ & 3 & 1 & 180,36 & 541,08 & $1.352,70$ & 3 & \begin{tabular}{|l|l|} 
MFP \\
\end{tabular} & MFP & $\mathrm{Em+H}$ & $\mathrm{FH}$ & $\mathrm{Ea}+\mathrm{H}$ & NO & 2.2 & 1 \\
\hline & 5405 & $44-40$ & 3 & 1 & 132,66 & 397,98 & 994,95 & 3 & \begin{tabular}{|l} 
MFP \\
\end{tabular} & MFP & $\mathrm{Em}+\mathrm{H}$ & $\mathrm{FH}$ & $\mathrm{Ea}+\mathrm{H}$ & NO & 2.2 & 1 \\
\hline & 5406 & 38 & 0 & 1 & 274,57 & & & Solar & MFP & MFP & $E m+H$ & $\mathrm{FH}$ & $\mathrm{Ea}+\mathrm{H}$ & NO & 2.2 & 1 \\
\hline & $\frac{5407}{508}$ & $36-34$ & 3 & 1 & 111,03 & 333,09 & 832,73 & 3 & \begin{tabular}{|l|l|} 
MFP \\
\end{tabular} & MFP & Em+H & $\mathrm{FH}$ & $\mathrm{FHI}$ & NO & 2.3 & 1 \\
\hline S4 & 5408 & $32-30$ & 3 & 1 & $\begin{array}{ll}108,61 \\
\end{array}$ & 325,83 & 814,58 & 3 & \begin{tabular}{|l|l|} 
MFP \\
\end{tabular} & MFP & $\mathrm{Em}+\mathrm{H}$ & $\mathrm{FH}$ & $\mathrm{FHI}$ & NO & 2.3 & 1 \\
\hline & $\frac{5409}{540}$ & $28-26$ & 2 & 1 & 60,31 & 120,62 & 301,55 & 2 & \begin{tabular}{|l|l|} 
MFP \\
\end{tabular} & MFP & Em+H & $\mathrm{FH}$ & $\mathrm{FHI}$ & NO & 2.3 & 1 \\
\hline & $\begin{array}{l}5410 \\
5411\end{array}$ & $\frac{24}{22-20}$ & $\frac{2}{3}$ & $\frac{1}{1}$ & $\begin{array}{r}55,98 \\
119,20 \\
\end{array}$ & $\begin{array}{l}111,96 \\
357,60\end{array}$ & $\frac{279,90}{894,00}$ & $\frac{2}{3}$ & \begin{tabular}{|l|} 
MFP \\
MFP \\
\end{tabular} & $\begin{array}{l}\text { MFP } \\
\text { MEP }\end{array}$ & $\left|\frac{E m+H}{E m+H}\right|$ & \begin{tabular}{|l|}
$F H$ \\
$F H$ \\
\end{tabular} & \begin{tabular}{|l}
$\mathrm{Ea}+\mathrm{H}$ \\
$\mathrm{E}+\mathrm{H}$
\end{tabular} & $\begin{array}{l}\text { NO } \\
\text { NO }\end{array}$ & $\frac{2.2}{2.2}$ & $\frac{1}{1}$ \\
\hline & $\mathrm{s} 412$ & $18-14$ & 3 & 1 & 171,27 & 513,81 & $1.284,53$ & 3 & MFP & MFP & $\mathrm{Em}+\mathrm{H}$ & $\mathrm{Em}+\mathrm{H}$ & $E$ & \begin{tabular}{|l|} 
Estructura histórica \\
singular Palacete
\end{tabular} & 2.1 & 1 \\
\hline & 5413 & 12B-10 & 2 & 1 & 103,35 & 206,70 & 516,75 & 2 & \begin{tabular}{|l|l|} 
MFP \\
\end{tabular} & MFP & $\mid$ & FH & \begin{tabular}{|l|}
$\mathrm{FHII}$ \\
\end{tabular} & NO & 2.3 & 2 \\
\hline & 5414 & 8 & 3 & 1 & & 123,66 & 309 & 3 & \begin{tabular}{|l|l|} 
MFP \\
\end{tabular} & MFP & $\mathrm{Em}+\mathrm{H}$ & \begin{tabular}{|l|l|}
$F H$ \\
\end{tabular} & $\mathrm{Ea}+\mathrm{H}$ & NO & 2.2 & 7 \\
\hline & 5415 & 6 & 2 & 1 & 44,16 & 88,32 & 220,80 & 2 & MFP & MFP & $\mathrm{Em}+\mathrm{H}$ & $\mathrm{FH}$ & $\mathrm{Ea}+\mathrm{H}$ & NO & 2.2 & 3 \\
\hline & 5416 & $2-4$ & 3 & 1 & 115,34 & 346,02 & 865,05 & 3 & $\mathrm{MF}$ & $\mathrm{P}$ & $\mathrm{FH}$ & $\mathrm{FH}$ & $\mathrm{FH}$ & Edificio reciente & 3 & 3 \\
\hline S5 & 5501 & - & 3 & 1 & 205,29 & 615,87 & $1.539,68$ & 3 & \begin{tabular}{|l|}
$\mathrm{MF}$ \\
\end{tabular} & $P$ & $\mathrm{FH}$ & $\mathrm{FH}$ & $\mathrm{FH}$ & Edificio reciente & 3 & 3 \\
\hline & N101 & 159 & 3 & 1 & 171,69 & 515,07 & $1.287,68$ & 3 & \begin{tabular}{|l|l|} 
MFP \\
\end{tabular} & MF & $\mathrm{FH}$ & $\mathrm{FH}$ & $E$ & NO & 2.1 & 3 \\
\hline & N102 & 157 & 3 & 1 & 176,83 & 530,49 & $1.326,23$ & 3 & \begin{tabular}{|l|} 
MFP \\
\end{tabular} & $\mathrm{MF}$ & $\mathrm{FH}$ & $\mathrm{FH}$ & $E$ & NO & 2.1 & 3 \\
\hline & $\mathrm{N} 103$ & 155 & 3 & 1 & 113,82 & 341,46 & 853,65 & 3 & \begin{tabular}{|l|l|} 
MFP \\
\end{tabular} & $\mathrm{MF}$ & $\mathrm{FH}$ & $\mathrm{FH}$ & $E$ & NO & 2.1 & 3 \\
\hline N1 & N104 & 153-151 & 3 & 1 & 111,74 & 335,22 & 838,05 & 3 & \begin{tabular}{|l|l|} 
MFP \\
\end{tabular} & MF & $\mathrm{FH}$ & $\mathrm{FH}$ & $E$ & NO & 2.1 & 3 \\
\hline IV 1 & N105 & 149-143 & 4 & 1 & 346,62 & $1.386,48$ & $3.466,20$ & $5 S+3$ & \begin{tabular}{|l|l|} 
MFP \\
\end{tabular} & MF & $\mathrm{FH}$ & $\mathrm{FH}$ & $E$ & NO & 2.1 & 3 \\
\hline & N106 & 141-133 & 3 & 1 & 439,91 & $1.319,73$ & $3.299,33$ & 3 & \begin{tabular}{|l|} 
MFP \\
\end{tabular} & $\mathrm{MF}$ & \begin{tabular}{|l|}
$F H$ \\
\end{tabular} & $\mathrm{FH}$ & $E$ & NO & 2.1 & 3 \\
\hline & $\begin{array}{ll}\text { N107 } \\
\end{array}$ & \begin{tabular}{|l|}
$131-127$ \\
\end{tabular} & 3 & 1 & 166,44 & 499,32 & $1.248,30$ & 3 & \begin{tabular}{|l|l|} 
MFP \\
\end{tabular} & MF & \begin{tabular}{|l|l|}
$\mathrm{FH}$ \\
\end{tabular} & $\mathrm{FH}$ & FHI? & NO & 2.3 & 1 \\
\hline & \begin{tabular}{|l|l|} 
N108 \\
\end{tabular} & 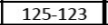 & 3 & 1 & $\begin{array}{ll}110,53 \\
\end{array}$ & 331,59 & 828 & 3 & MFP & MF & $\mathrm{FH}$ & $F H$ & $\mathrm{E}+\mathrm{H}$ & NO & 2.3 & 1 \\
\hline & N201 & \begin{tabular}{|l|}
$121-115$ \\
\end{tabular} & 3 & 1 & 311,17 & 933,51 & $2.333,78$ & 3 & MFP & MF & $E+H$ & $\mathrm{FH}$ & FHI & NO & 2.2 & 3 \\
\hline N2 & N202 & 113 & 3 & 1 & 70,31 & 210,93 & 527,33 & 3 & MFP & MFP & $E+H$ & $E+H$ & E & $\begin{array}{l}\text { Estructura histórica } \\
\text { singular Palacecte }\end{array}$ & $2.1 / 1$ & 1 \\
\hline 102 & N203 & 111-105 & 3 & 1 & 212,60 & 637,80 & $1.594,50$ & 3 & \begin{tabular}{|l|} 
MFP \\
\end{tabular} & MF & \begin{tabular}{|l|}
$\mathrm{E}+\mathrm{H}$ \\
\end{tabular} & \begin{tabular}{|l|}
$E+H$ \\
\end{tabular} & $E$ & NO & 2.2 & 1 \\
\hline & N204 & 103-93 & 3 & 1 & 329,90 & 989,10 & $2.4 / 4,23$ & 3 & \begin{tabular}{|l|l}
$M H P$ \\
\end{tabular} & $\mathrm{Mr}$ & \begin{tabular}{|l|l|}
$+H$ \\
\end{tabular} & $\mathrm{FH}$ & $\mathrm{FHI}$ & $\mathrm{NO}$ & 2.3 & 2 \\
\hline N3 & N301 & - & 4 & 1 & 25,33 & 101,32 & 253,30 & 6 torre & MFP & MFP & $\mathrm{E}$ & $\mathrm{E}$ & $\mathrm{E}$ & $\begin{array}{l}\text { ricica singular } \\
\text { torre }\end{array}$ & 1 & 3 \\
\hline & N400 & & 1,4 & 1 & 368,11 & 515,35 & $1.288,38$ & Equiv a 3 & MFP & MFP & $\bar{E}$ & & $E+H$ & \begin{tabular}{|c|} 
Histórica singular \\
iglesia
\end{tabular} & 2.1 & 2 \\
\hline & N401 & $91-79$ & 4 & 1 & 355,66 & $1.422,64$ & $3.556,60$ & 4 & \begin{tabular}{|l|} 
MFP \\
\end{tabular} & MFP & $\mathrm{Em}+\mathrm{H}$ & $\mathrm{FH}$ & $\mathrm{Ea}+\mathrm{H}$ & $\begin{array}{l}\text { NO } \\
\text { NO }\end{array}$ & 2.3 & 1 \\
\hline & N402 & 77 & 4 & 1 & 54,11 & 216,44 & 541,10 & 4 & \begin{tabular}{|l|} 
MFP \\
\end{tabular} & MFP & $\mathrm{Em}+\mathrm{H}$ & $\mathrm{FH}$ & $\mathrm{Ea}+\mathrm{H}$ & NO & 2.3 & 1 \\
\hline & $\mathrm{N} 403$ & 75-71 & 3 & 1 & $\begin{array}{l}120,23 \\
\end{array}$ & & & 3 & \begin{tabular}{|l|l|} 
MFP \\
\end{tabular} & MFP & $\mathrm{Em}+\mathrm{H}$ & $\mathrm{FH}$ & $\mathrm{Ea}+\mathrm{H}$ & NO & 2.3 & 1 \\
\hline & N404 & & 3 & 1 & $\begin{array}{l}54,49 \\
\end{array}$ & & 408 & 3 & \begin{tabular}{|l|} 
MFP \\
\end{tabular} & MFP & $\mathrm{Em}+\mathrm{H}$ & $\mathrm{FH}$ & $\mathrm{Ea}+\mathrm{H}$ & $\mathrm{NO}$ & 2.3 & 1 \\
\hline & N405 & $65-61$ & 3 & 1 & 167,72 & & 1.257 & 3 & \begin{tabular}{|l|l|} 
MFP \\
\end{tabular} & MFP & $\mathrm{Em}+\mathrm{H}$ & $\mathrm{FH}$ & $E$ & $\mathrm{NC}$ & 2.2 & 2 \\
\hline & N406 & 59 & 3 & 1 & 91,25 & & 68 & 3 & \begin{tabular}{|l|} 
MFP \\
\end{tabular} & MFP & $\mathrm{Em}+\mathrm{H}$ & $\mathrm{FH}$ & \begin{tabular}{|l|l|}
$\mathrm{Ea}+\mathrm{H}$ \\
\end{tabular} & $\mathrm{NC}$ & 2.3 & 1 \\
\hline & N407 & $57-$ & 3 & 1 & 196,78 & 590 & 1.47 & 3 & \begin{tabular}{|l|} 
MFP \\
\end{tabular} & MFP & $\mathrm{Em}+\mathrm{H}$ & $\mathrm{FH}$ & Ea+H & NO & 2.3 & 1 \\
\hline & N408 & $53-51$ & 3 & 1 & 68,36 & 205 & 512 & 3 & \begin{tabular}{|l|} 
MFP \\
\end{tabular} & MFP & $\mathrm{Em}+\mathrm{H}$ & $\mathrm{FH}$ & E & NO & 2.2 & 2 \\
\hline & N409 & $49-41$ & 3 & 1 & 223,67 & 671,01 & $1.677,53$ & 3 & \begin{tabular}{|l|} 
MFP \\
\end{tabular} & MFP & $\mathrm{Em}+\mathrm{H}$ & \begin{tabular}{|l|l|}
$F H$ \\
\end{tabular} & $E$ & NO & 2.2 & 2 \\
\hline N4 & N410 & 39 & 3 & 1 & 64,96 & 194,88 & $\begin{array}{r}487,20 \\
\end{array}$ & 3 & \begin{tabular}{|l|l|} 
MFP \\
\end{tabular} & MFP & $\mathrm{Em}+\mathrm{H}$ & $\mathrm{FH}$ & \begin{tabular}{|l|l|}
$\mathrm{FH}$ \\
\end{tabular} & NO & 2.3 & 1 \\
\hline 104 & N411 & $37-33$ & 3 & 1 & 146,81 & 440,43 & $1.101,08$ & 3 & \begin{tabular}{|l|} 
MFP \\
\end{tabular} & MFP & $\mathrm{Em}+\mathrm{H}$ & \begin{tabular}{|l|}
$F H$ \\
\end{tabular} & \begin{tabular}{|l|l|}
$\mathrm{FH}$ \\
\end{tabular} & NO & 2.3 & 1 \\
\hline & $\mathrm{N} 412$ & 31 & 3 & 1 & 53,16 & 159,48 & 398 & 3 & \begin{tabular}{|l|} 
MFP \\
\end{tabular} & MFP & $\mathrm{Em}+\mathrm{H}$ & $\mathrm{FH}$ & $E$ & $\mathrm{NC}$ & 2.2 & 3 \\
\hline & N413 & 29 & 3 & 1 & 66,50 & 199,50 & 498 & 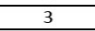 & \begin{tabular}{|l|} 
MFP \\
\end{tabular} & MFP & $\mathrm{Em}+\mathrm{H}$ & 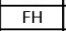 & $E$ & $\mathrm{~N}$ & 2. & 3 \\
\hline & N414 & 27 & 3 & 1 & 75,46 & 226,38 & 565, & 3 & \begin{tabular}{|l|l|} 
MFP \\
\end{tabular} & MFP & Em+H & $\mathrm{FH}$ & $E$ & $\mathrm{~N}$ & 2.2 & 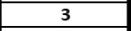 \\
\hline & $\mathrm{N} 41$ & 25 & 3 & 1 & 96,97 & 290,9 & 727 & 3 & \begin{tabular}{|l|} 
MFP \\
\end{tabular} & MFP & $\mathrm{Em}+\mathrm{H}$ & $\mathrm{FH}$ & $E$ & $\mathbb{N}$ & & 2 \\
\hline & N416 & 23 & 3 & 1 & 51,08 & 15 & 38 & 3 & MFP & MFP & $\mathrm{Em}+\mathrm{H}$ & $\mathrm{FH}$ & $E$ & $\sqrt{N}$ & 2.2 & 2 \\
\hline & & 21- & 3 & 1 & & 19 & & 3 & & MFP & & $\mathrm{H}$ & $E$ & $\mathrm{~N}$ & 2.2 & 2 \\
\hline & & 1 & 3 & 1 & & & & 3 & MFP & MFP & Em+H & $\mathrm{H}$ & $\mathrm{Ea}+\mathrm{H}$ & $\mathrm{N}$ & 2.3 & 1 \\
\hline & & $15-11$ & 2 & 1 & & 295 & 738 & 2 & MFP & MFP & Em+H & $\mathrm{H}$ & $E$ & $\mathrm{~N}$ & 2.2 & 3 \\
\hline & N420 & 7 & 3 & 1 & 201,94 & 605,82 & $1.514,55$ & 3 & \begin{tabular}{|l|} 
MFP \\
\end{tabular} & MFP & $\mathrm{Em}+\mathrm{H}$ & $\mathrm{FH}$ & E & NO & 2.2 & 2 \\
\hline & N421 & $5-1$ & 3 & 1 & 76,96 & 230,88 & 577,20 & 3 & \begin{tabular}{|l|} 
MFP \\
\end{tabular} & MFP & $\mathrm{Em}+\mathrm{H}$ & $\mathrm{FH}$ & $E$ & NO & 2.2 & 3 \\
\hline & N501 & - & & 1 & 450,00 & - & - & Equiv a 4. & MFP & MFP & E & & $\mathrm{E}$ & $\begin{array}{c}\text { itstricas aningular } \\
\text { iglessia }\end{array}$ & 1 & 2 \\
\hline N5 & N502 & - & 4 & 1 & 25,33 & 101,32 & 253,30 & 6 torre & MFP & MFP & $E$ & $E$ & $E$ & \begin{tabular}{|l|} 
Histórica singular \\
torre
\end{tabular} & 1 & 3 \\
\hline & N503 & & 1 & 0,6 & 39,01 & 39,01 & 58,51 & 2 & \begin{tabular}{|l|l|} 
MFP \\
\end{tabular} & MFP & $\mathrm{B}+\mathrm{H}$ & & & si, Puerta ciudad & 2.1 & 3 \\
\hline
\end{tabular}

Abreviaturas utilizadas en la toma de datos: MFP, muro de fábrica de piedra. MFL, muro de fábrica de ladrillo. MF, muro de fábrica indeterminado. P, pilares de hormigón/h.a. E, entramado. FH, forjado de hormigón, normalmente con vigas de acero, aunque sea precario. FHI, forjado inclinado de hormigón. E, entramado no monolítico. Ea, entramado de perfiles de acero no monolítico. Em, entramado de madera. E+H, entramado y capa de hormigón añadida. Variaciones con respecto a esta tipología se indicaron en observaciones. 
TIPO DE ESTRUCTURA DAÑOS ESTRUCTURALES

ALTERACIONES

SOBRE LA ESTRUCTURA ORIGINAL

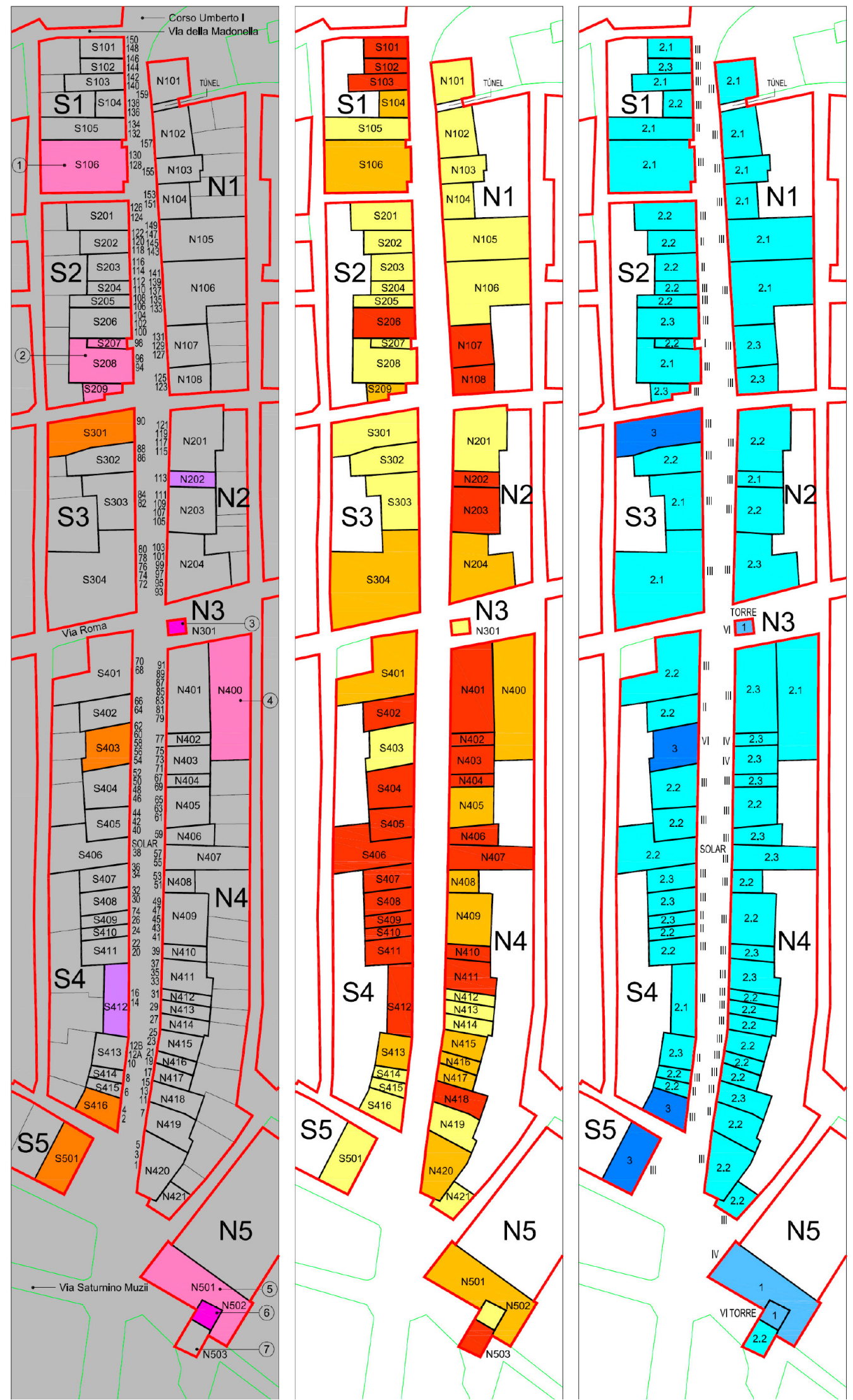

TIPO DE ESTRUCTURA

ESTRUCTURAS

HISTÓRICAS SINGULARES

ESBELTAS (TIPO TORRE) TIPO DIÁFANO

TIPO DIÁFAN
(IGLESIA)

DE PISOS
(PALACETES

ESTRUCTURAS

RECIENTES COMPLETAS

DE PISOS (MENOS 60 AÑOS)

RESTO DE ESTRUCTURAS

HISTORICAS POPULARES
O BURGUESAS (PRIMERA MITAD $s . X X$

(1) Centro cultural

Consiglio Comunale.

(Iglesia de S. Giuseppe)

(2) Antigua Iglesia

(3) Torre Cívica

4) Iglesia de S. Giovanni.

5) Iglesia de S. Agostino.

6) Torre de la iglesia de

S. Agostino.

(7) Porta Carbonara.

DAÑOS ESTRUCTURALES DAÑOS REGISTRADOS

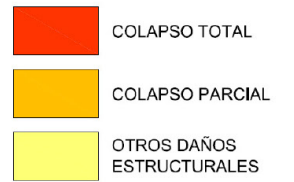

ALTERACIONES SOBRE LA ESTRUCTURA ORIGINAL TIPO 1: ESTRUCTURAS HISTÓRICAS NO ALTERADAS

\section{ESBELTAS
(TIPO TORRE)}

TIPO 2: ESTRUCTURAS HISTÓRICAS ALTERADAS

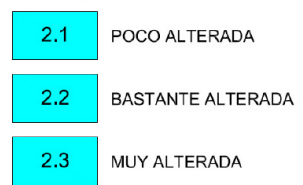

TIPO 3: ESTRUCTURAS RECIENTES (NO ALTERADAS)

3 DE PISOS DE PISOS
(MENOS 67 AÑOS)

Figura 5. Plano resumen de resultados. 


\section{DISCUSIÓN. DIAGNÓSTICO DE DAÑOS}

\subsection{Constatación de hechos}

Los resultados expuestos constatan lo siguiente:

- Existencia de un gran número de estructuras alteradas.

Lo primero que se constató in situ en el análisis forense efectuado de los restos, fue el gran número de edificios cuyas estructuras originales habían sido profundamente alteradas, empleando materiales distintos a los de la estructura original (véanse figuras 6 y 7) y mixtificando los esquemas originales de funcionamiento estructural. Del total de 74 edificios de la zona de estudio, sólo 7 (9,46\%) no tenían alteradas sus estructuras. El resto (90,54\%) tenían alteradas sus estructuras originales en distinta medida.

Estos datos contrastan con la protección legal-patrimonial que tenían los edificios del centro histórico y demuestran que, aunque se haya mantenido una protección "ambiental" y una protección mayor de determinados edificios singulares, la mayoría de los edificios se habían protegido sólo "en sus aspectos formales exteriores", mientras eran intensamente alteradas sus estructuras resistentes por el interior, para ampliaciones en altura, ampliaciones en ocupación de parcela, reformas interiores, cambios de uso por ejemplo en los locales comerciales de planta baja, etc.

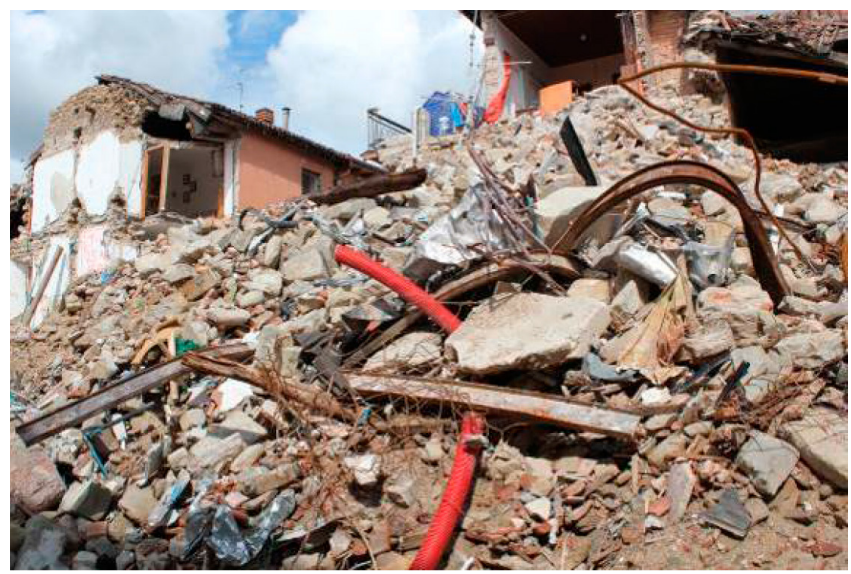

Figura 6. La existencia de nuevos materiales estructurales ha sido fácilmente constatable en los restos de los derrumbes.

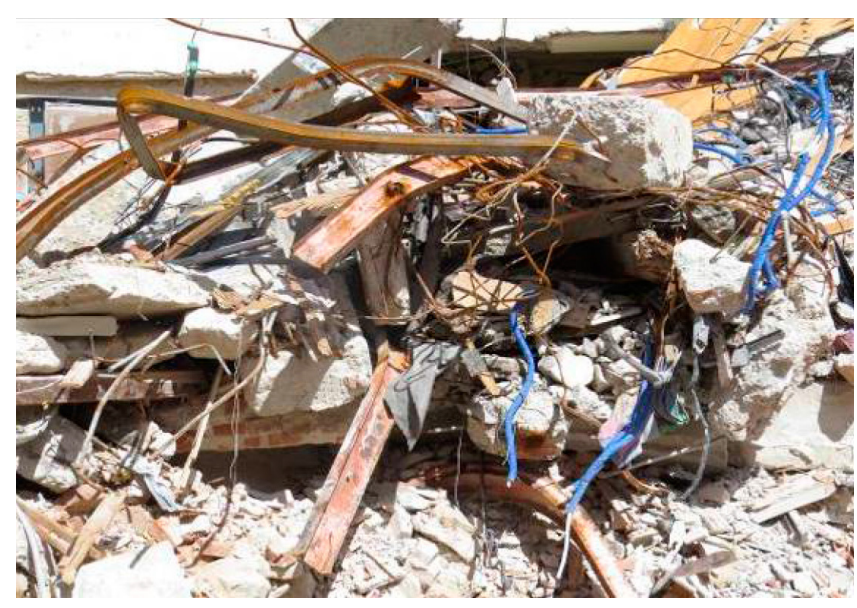

Figura 7. Obsérvense perfiles de acero laminado y restos de hormigón armado, materiales todos ellos ajenos a la estructura histórica, presuntamente "reforzada" con materiales nuevos.
- Los tipos estructurales mixtos detectados no están incluidos en las normas sísmicas.

La segunda constatación es que los sistemas estructurales complejos resultado de las alteraciones, encontrados en los restos de los escombros (que afectan a dicho 90,54\%), no son los tipos estructurales "simplificados" que aparecen en la norma sísmica europea (Eurocódigo-8) (5) y en la EMS-98 (9) (que sólo considera estructuras de fábrica y estructuras de hormigón armado), sino otros de distinta caracterización, de funcionamiento estructural también distinto y que se encontraban en un limbo legal.

\subsection{Terremoto muy destructivo}

Forma parte del estado del conocimiento (10) que el riesgo sísmico depende tanto de la peligrosidad sísmica de una región (la aceleración sísmica que se produce en caso de terremoto) como de la vulnerabilidad sísmica de los edificios. En la zona de Amatrice, el mayor riesgo sísmico se ha dado en el centro histórico, pues aunque todo el área tiene la misma peligrosidad sísmica, los edificios históricos y, especialmente los históricos con estructuras alteradas, han demostrado ser los más vulnerables (vulnerabilidad sísmica).

El terremoto ha sido muy destructivo para una potencia de magnitud Mw de 6,2, tanto por la gran acción sísmica incidente (aceleración causada a las construcciones por tener su hipocentro a escasa profundidad, sólo 4,4 km), como por la vulnerabilidad sísmica de las construcciones ahora constatada.

\subsection{Los mayores daños, en edificios con estructuras alteradas}

\section{- Peor comportamiento de las estructuras alteradas.}

El contraste entre los planos segundo y tercero acredita que los edificios que menos daños han registrado son aquellos que habían tenido una menor alteración sobre la estructura original, ya fuera ésta histórica o reciente, lo que constituye la aportación fundamental de este trabajo de investigación.

Se comprueba que ninguno de los tipos 1 (estructuras históricas no alteradas) ni 3 (estructuras recientes, no alteradas) ha registrado colapsos totales. Véase la tabla 7, de daños estructurales según tipos de estructuras:

Tabla 7. Daños estructurales según tipos de estructuras.

\begin{tabular}{|c|c|c|c|c|}
\hline \multirow[b]{2}{*}{ TIPO } & \multirow{2}{*}{$\begin{array}{l}\text { PARCELA/ } \\
\text { EDIFICIO }\end{array}$} & \multicolumn{3}{|c|}{ DAÑOS ESTRUCTURALES } \\
\hline & & $\begin{array}{c}\text { Colapso } \\
\text { total }\end{array}$ & $\begin{array}{c}\text { Colapso } \\
\text { parcial }\end{array}$ & $\begin{array}{l}\text { Otros } \\
\text { daños }\end{array}$ \\
\hline \multirow{4}{*}{$\begin{array}{l}\text { TIPO } 3 \\
\text { Estructuras } \\
\text { recientes, no } \\
\text { alteradas. }\end{array}$} & S301 & NO & NO & Sí \\
\hline & $\begin{array}{l}\text { S403. Edificio } \\
\text { denominado } \\
\text { "rojo". }\end{array}$ & NO & NO & Sí \\
\hline & S416. & NO & NO & SÍ \\
\hline & S501. & NO & NO & Sí \\
\hline \multirow{3}{*}{$\begin{array}{l}\text { TIPO } 1 \\
\text { Estructuras } \\
\text { históricas no } \\
\text { alteradas. }\end{array}$} & $\begin{array}{l}\text { N301. Torre } \\
\text { Cívica. }\end{array}$ & NO & NO & Sí \\
\hline & $\begin{array}{l}\text { N501. Iglesia de } \\
\text { S. Agostino. }\end{array}$ & NO & NO & Sí \\
\hline & $\begin{array}{l}\text { N502. Torre de } \\
\text { la iglesia de S. } \\
\text { Agostino. }\end{array}$ & NO & Sí & Sí \\
\hline
\end{tabular}


Tampoco ninguna de estas estructuras ha registrado colapsos parciales, a excepción de la iglesia de S. Agostino que vio colapsado su hastial. Sin embargo, este colapso es "de manual" (13).

Merece también comentario la iglesia de S. Giovanni, que había sido alterada en su estructura (con una capa de hormigón en su entramado de madera de cubierta y unos "refuerzos" de acero en sus cerchas de cubierta), registrando daños superiores a los normales (superiores a los de la iglesia de S. Agostino, de similar estructura).

- Digno comportamiento de las estructuras históricas no alteradas.

De las 4 estructuras históricas singulares diáfanas (tipo iglesia):

- Ninguna ha registrado colapso total.

- 3 han registrado colapsos parciales (que representan el 75,00\%): S106 (antigua iglesia de S. Giuseppe), N400 (iglesia de S. Giovanni) y N501 (iglesia de S. Agostino).

- 1 ha registrado otro tipo de daños estructurales (el 25,00\%): S208.

Ha influido en el balance de daños de estas construcciones, la vulnerabilidad sísmica de los hastiales de fachada, ya enunciada por Lagomarsino (1), característicos de la arquitectura medieval italiana, que con su gran masa, vuelcan, colapsando parcialmente la fachada y arrastrando normalmente a la primera crujía de cubierta. Esta forma de colapso se ha observado tanto en N40o (iglesia de S. Giovanni) como en N501 (iglesia de S. Agostino). La misma forma de colapso se ha podido apreciar también en el terremoto de Lorca (13).

De las 2 estructuras históricas singulares tipo esbelto (torres): N301 (torre Cívica) y N5O2 (torre de la iglesia de S. Agostino).

- Ninguna ha registrado colapso total o colapso parcial.

- Las 2 han registrado otro tipo de daños estructurales (el 100,00\%): Llamó poderosamente la atención desde el primer momento, cómo era posible que las torres, que debido a su esbeltez son el tipo de construcción más vulnerable a una acción horizontal de sismo, permanecieran en pie tras un terremoto que había producido el arrasamiento del centro histórico. Encontramos la explicación en la no alteración de su estructura histórica (véanse figuras 8 y 9).

Otros autores habían comparado los daños estructurales del terremoto de 2011 en Amatrice y en el núcleo próximo de Norcia (14), llegando a la conclusión de que los daños en Norcia fueron menores porque esta ciudad había sido reforzada tras el terremoto anterior de 1860 , pero sin haber considerado que en la actualidad Norcia carece de un centro histórico de las características de vulnerabilidad de Amatrice. Por el contrario, tras la presente investigación estamos más de acuerdo con Sorrentino (15), que compara sólo las torres-campanario de estos dos núcleos de población, para concluir que en la secuencia de terremotos entre agosto de 2016 y enero de 2017 , las torres sí se derrumbaron en Norcia y no en Amatrice.

De las estructuras históricas singulares de tipo pisos (palacetes):

- Las 2 han registrado colapso total (el 100,00\%): N2O2 y S412.
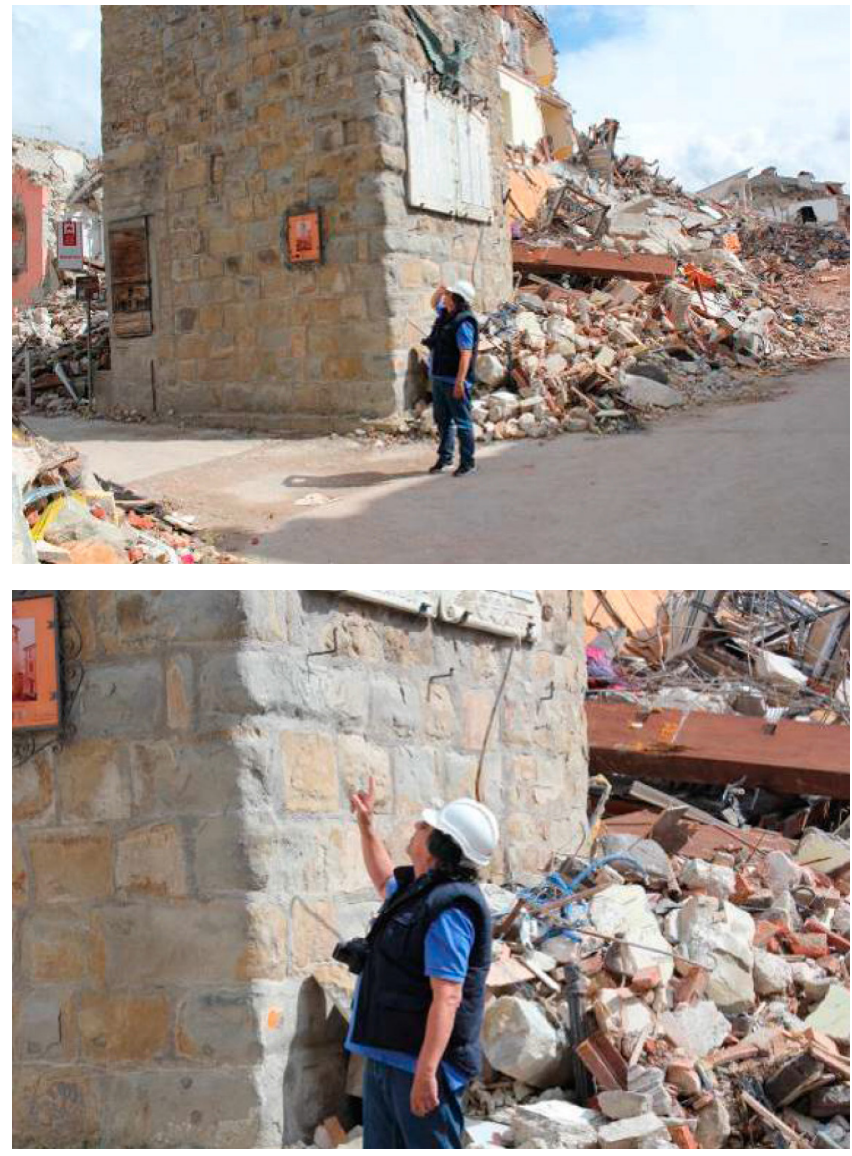

Figuras 8 y 9. Con sólo fábrica de piedra y mortero de cal, la torre Cívica, del s. XIII (N301) y la torre de la iglesia de S. Agostino, del s. XV (N502), es decir, todas las torres del área de estudio (que son el tipo estructural más vulnerable a un sismo) contemplan desde su altura el arrasamiento del centro histórico.

Se han detectado en los restos de ambos edificios, multitud de añadidos de hormigón, tales como capas de hormigón sobre los entramados y (en N202) incluso "refuerzos" de los muros en su intradós, con mallazos de acero y capas de mortero que pretendían darles "más resistencia".

Como conclusión de las estructuras históricas singulares de todos los tipos (iglesias, torres y palacetes):

- 2 han registrado colapso total (el 25,00\%).

- 3 han registrado colapsos parciales (el 37,50\%).

- 3 han registrado otro tipo de daños estructurales (el $37,50 \%)$.

- Conocido comportamiento de las estructuras recientes (no alteradas).

De los 4 edificios de nueva planta recientes con estructura de pisos (completa de forjados de hormigón armado y pilares desde cimentación):

- Ninguno ha registrado colapso total o colapso parcial.

- Los 4 han registrado otro tipo de daños estructurales (el 100,00\%): S3O1 (de ladrillo visto amarillo, de la C/ Umberto I, $\mathrm{n}^{\mathrm{O}}$ 90), $\mathrm{S} 4 \mathrm{O} 3$ (de ladrillo visto rojo, de la C/ Umberto I, $n^{0}$ 54-62), S416 y S501. Véase figura 10.

Es decir, que todos ellos se han mantenido en pie permitiendo el desalojo de las personas que los ocupaban, protegiéndo- 


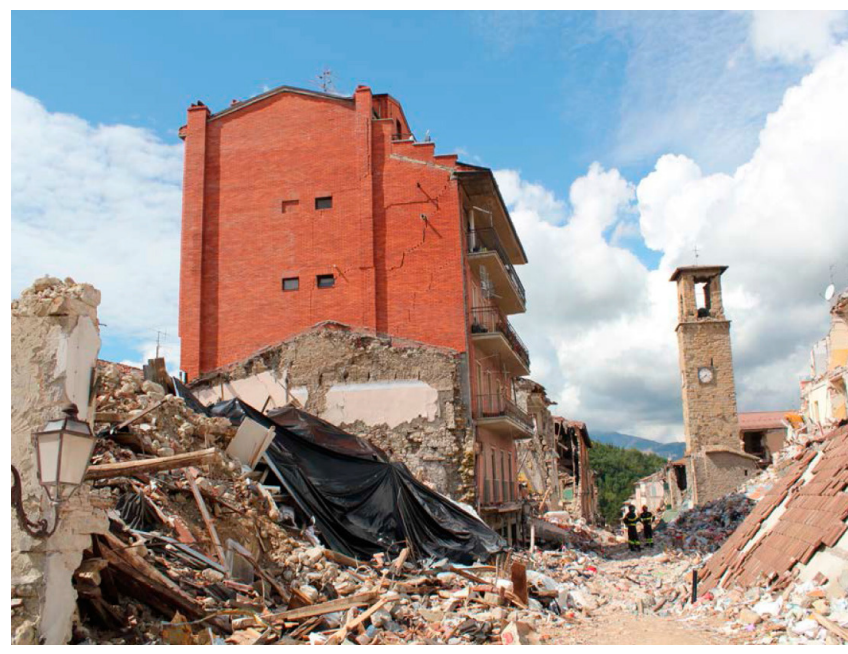

Figura 10. Esta impactante imagen muestra cómo dos edificios, uno histórico (la torre Cívica) y otro reciente (S403) conocido como "el edificio rojo", son casi los únicos en mantenerse en pie del centro histórico, pese a que la esbeltez es un punto negativo en la vulnerabilidad sísmica. Ambos edificios coinciden en una cosa: que sus estructuras eran las originales y no habían sido alteradas.

los de daños personales. El hecho de que todos ellos hayan tenido luego que ser derribados (constatado por los autores en una nueva visita en 2018) tiene que ver con que la reparación de los daños que presentaban resultara inviable o demasiado costosa en contraste con el valor (no patrimonial histórico) de estos inmuebles.

\section{- El grupo de los edificios más dañados.}

Una vez explicadas las estructuras históricas singulares y las estructuras recientes, se entra ahora a la evaluación de daños del resto de edificios, que son precisamente los que habían sido más intensamente alterados en sus estructuras originales.

De este resto de construcciones con estructura de pisos, históricas-populares o racionalistas, de construcción hasta los años 50 del siglo XX (aunque puedan haber registrado recientes ampliaciones y/o reformas y/o cambios de uso):

- 25 han registrado colapso total (el 41,67\%).

- 13 han registrado colapsos parciales (el 21,67\%).

- 22 han registrado otro tipo de daños estructurales (el $36,67 \%)$.

En su forma de colapso ha influido la alteración de sus estructuras originales con añadidos de hormigón (pesados y rígidos) que han podido ser bien identificados en la inspección forense de los restos (figuras 11 y 12).

\subsection{Sobre los modelos estructurales y futuras líneas de investigación.}

La estructura debe ser estudiada en su conjunto, es una obviedad que conviene recordar. El hecho de que se puedan realizar en la actualidad estructuras más seguras con hormigón armado completas, no quiere decir que interviniendo con estas técnicas sólo parcialmente sobre estructuras históricas preexistentes se vaya a conseguir mayor seguridad estructural.

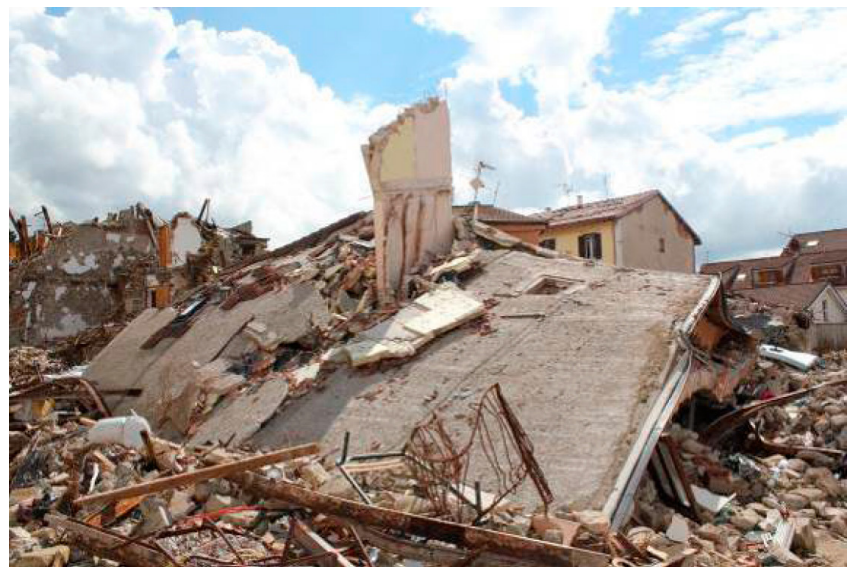

Figura 11. Ejemplo de estructura de hormigón en faldones monolíticos de cubierta, añadida en una segunda planta sobre un edificio histórico de muros. Se ha producido el desequilibrio por vuelco.

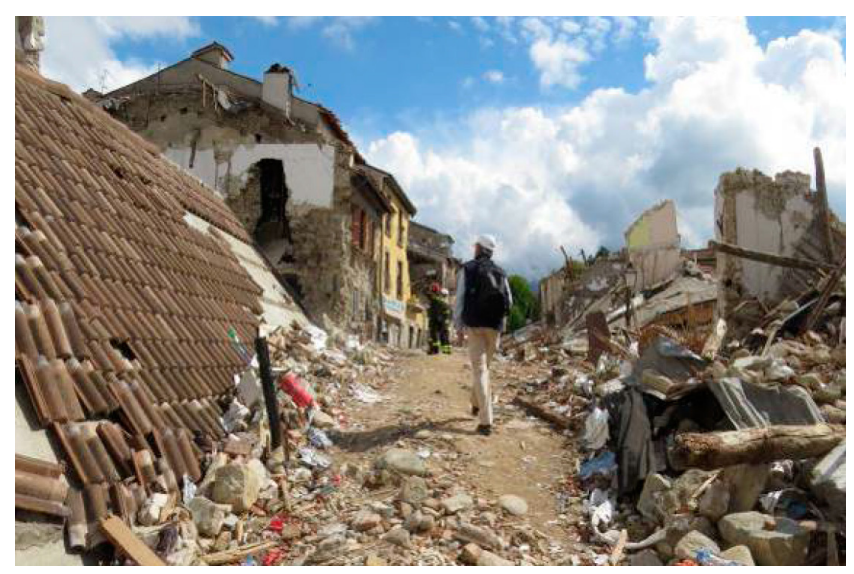

Figura 12. En este otro ejemplo de la misma forma de colapso, el faldón de cubierta de hormigón armado desequilibrado, incluso ha mantenido las tejas en su sitio, pero las dos plantas de muros en las que se apoyaba han "desaparecido" por la acción producida por la masa, rigidez y pendiente del forjado.

Lagomarsino, que en 2012, a partir de los estudios del terremoto de L'Aquila (2009) apreció por primera vez "el mal comportamiento de las iglesias reforzadas por técnicas modernas, como la sustitución de techos de madera originales con rígidos y pesados de losas de hormigón armado" (1) y valoró la complejidad de enfrentarse al fenómeno de las estructuras mixtificadas con un modelo estructural; sin embargo, en trabajos posteriores (16), ha investigado con otros autores sobre hipótesis y modelos de cálculo, también para el caso de edificios monumentales. Pero los hechos demuestran que edificios históricos reforzados en la zona de estudio después del terremoto de L'Aquila (2009), como la escuela infantil de Amatrice, han colapsado de forma estrepitosa tras el terremoto de 2016.

Es necesario ir a los fundamentos del diseño estructural. Acudiendo a Eduardo Torroja, "el cálculo no es más que una herramienta para prever si las formas y dimensiones de una construcción (...) son aptas para soportar las acciones a que ha de estar sometida" (11), "la obra no nace nunca del cálculo, es el cálculo el que resulta del diseño de la estructura, el que pospuesto a él, se encarga de garantizar las condiciones de estabilidad y resistencia...” (11) y los modelos estructurales son meras simplificaciones de la realidad para poder llegar a conclusiones de 
proyecto (o de peritaje en el caso que nos ocupa) (11). Lo dice también el Código Técnico de la Edificación (17), el modelo es la "idealización del sistema estructural utilizada para el análisis, cálculo y verificación”. Por lo tanto, no tiene sentido insistir en hipótesis y en modelos que son desmentidos por el comportamiento estructural real, que es lo que viene ocurriendo en los terremotos que afectan a centros históricos intensamente alterados en sus estructuras originales.

Mientras se consigue un modelo adecuado para las estructuras históricas ante las acciones que las hacen más vulnerables, los investigadores que suscribimos esta investigación, abogamos por el buen conocimiento de la estructura histórica y por la evaluación cualitativa, que son las directrices dadas por ICOMOS (18) y admitidas por la normativa española (17) que en el Código Técnico de la Edificación dice que "En edificios en los que no resulte posible o sea poco fiable una verificación cuantitativa (...) podrá realizarse una evaluación cualitativa de la capacidad portante y de la aptitud al servicio" (Anejo D, apartado 2.1.2).

Las nuevas investigaciones para llegar a modelos fiables, deberían basarse en el estudio in situ y sobre casos reales de terremotos, para contrastar si las hipótesis de cálculo son adecuadas o no y si son aplicables o no a edificios de complejas estructuras mixtas de estructuras históricas y recientes. Es necesario además estudiar los terremotos inmediatamente tras el evento sísmico, con metodologías como la expuesta en este trabajo, pues las pruebas técnicas forenses desaparecen con la acción de los trabajos de rescate y con el desescombro de los restos de los terremotos.

Debido a la complejidad de las construcciones históricas, las actuales estrategias de la ingeniería sísmica no son directamente aplicables, como los sistemas de protección por disipación (empleados con buenos resultados en áreas de gran peligrosidad sísmica en edificios nuevos). Sin embargo las estrategias de resistencia y sobre todo la de ductilidad sí pueden ser experimentadas. También se recomienda recabar las estrategias históricas antisísmicas (19) para trabajar a partir de su análisis.

Se han producido avances en la investigación con la mejora de la resistencia al corte de las fábricas (20) y con las mejoras en los morteros estructurales para dotarles de ductilidad (21). Asimismo, en el diseño estructural de las intervenciones debe valorarse que los muros de mampostería no sólo proporcionan la resistencia a compresión para el peso, sino que, en caso de acciones horizontales, los muros proporcionan la estabilidad (resistencia lateral) con su comportamiento en el plano a las cargas cíclicas del sismo. Para ello la tensión vertical y las disposiciones de unión entre muros resultan determinantes (22).

\subsection{La extensión del fenómeno en los centros históricos}

El caso de Amatrice no es un caso aislado y las mixtificaciones estructurales constatadas por la alteración de las estructuras históricas originales son similares a las que se pueden encontrar en otros centros históricos patrimoniales del Sur de Eu- ropa. A juicio de los autores, esto es debido a la conjunción de tres políticas incompatibles entre sí:

- De protección del patrimonio histórico, que prohíben la destrucción de los muros de fábrica de mampostería.

- De urbanismo, que han permitido o consentido en general el aumento de altura de los edificios y la rehabilitación de los edificios de vivienda para otros usos.

- Y de "seguridad estructural", que propugnan estructuras de pisos de hormigón y de acero, cada vez más pesadas y más rígidas, pensando sólo en los edificios nuevos, sin tener en cuenta el comportamiento estructural del conjunto real que resulta de estas intervenciones.

Estas políticas han creado (sin pretenderlo) un sistema estructural mixto de muros de fábrica y pisos de hormigón, muy distinto estructuralmente, tanto del sistema histórico de fábrica (masonry structures), como de los sistemas estructurales recientes de hormigón de pilares y nudos rígidos. Y se ha constatado su pésimo comportamiento estructural en caso de sismo.

\section{CONCLUSIONES}

El diagnóstico estructural de los daños en el centro histórico patrimonial de Amatrice, producidos por el terremoto del 24 de agosto de 2016, de magnitud 6,2 Mw, con hipocentro a 4,4 $\mathrm{km}$ de profundidad, demuestra:

- Lo que ya era conocido en el estado de la técnica:

- Las estructuras históricas no alteradas han tenido un comportamiento estructural "de manual”, tratándose de construcciones vulnerables a los sismos.

- Las estructuras recientes, considerando como tales aquellas construidas de nueva planta con tecnologías de hormigón armado a partir de la segunda mitad del s. XX, han tenido un mejor comportamiento estructural que las anteriores con carácter general.

- La novedad del estudio realizado está:

- En la constatación del hecho de que más del 9o\% de los edificios del área representativa de estudio, tenían alteradas sus estructuras históricas originales.

- Y en la mayor vulnerabilidad sísmica que han registrado dichas estructuras alteradas con obras de ampliación, reforma, o cambios de uso, empleando materiales y técnicas recientes de hormigón y acero, monolíticas, que añadieron masa y rigidez a la estructura original.

Se ha evidenciado que las normas estructurales sísmicas (Eurocódigo-8, EMS-98...) no contemplan la rica complejidad de las estructuras de los edificios históricos, ni tampoco sus alteraciones cuando en ellos se ha intervenido con materiales y técnicas recientes de hormigón y acero. Pese a que estas tipologías están extendidas en los centros históricos.

Los daños registrados en el terremoto de Amatrice (6,2 Mw) en su centro histórico, son objetivamente superiores a los que recogen las escalas sísmicas para los "edificios de muros", para la intensidad del terremoto, de lo que se colige que interviniendo con hormigón y con acero en las estructuras de muros, se ha aumentado su vulnerabilidad sísmica. 


\section{REFERENCIAS}

(1) Lagomarsino, Sergio (2012). Damage assessment of churches after L'Aquila earthquake. Bulletin of Earthquake Engineering, 10: 73-92. https://doi.org/10.1007/s10518-011-9307-x

(2) Del Gaudio, C., Ricci, P. and Verderame, G. M. (2017). First remarks about the expected damage scenario following the 24th August 2016 earthquake in central Italy. En Proceedings of the 16th world conference on earthquake engineering.

(3) Salcedo Hernández, J.C. y Campesino Fernández, A.J. (2012). Experiencias constructivas del terremoto de Lorca. Investigaciones Geográficas, 57: 7-37. https://doi.org/10.14198/INGEO2012.57.01

(4) Zanini, Mariano-Angelo; Hofer, Lorenzo; Pellegrino, Carlo (2019). A framework for assessing the seismic risk map of Italy and developing a sustainable risk reduction program. International Journal of Disaster Risk Reduction, 33: 74-93. https://doi.org/10.1016/j.ijdrr.2018.09.012

(5) Eurocódigo-8 (2018). Proyecto para la resistencia al sismo para las estructuras. Comité Europeo para la Estandarización, Bruselas. UNE-EN 1998-1:2011: Reglas generales, acciones sísmicas y reglas para edificación, UNE-EN 1998-3:2018: Evaluación y adecuación sísmica de edificios.

(6) Stewart, Jonathan P., et al. (2018). Reconnaissance of 2016 Central Italy earthquake sequence. Earthquake Spectra, 34(4): 1547-1555. https://doi.org/10.1193\%2Fo80317EQS151M

(7) Grünthal, G., et al. (1999). Seismic hazard assessment for Central, North and Northwest Europe: GSHAP Region 3 , Annals of Geophysics, vol. 42(6): 999-1011. https://doi.org/10.4401/ag-3783

(8) Sorrentino, Luigi, et al. (2018). Seismic behaviour of ordinary masonry buildings during the 2016 central Italy earthquakes. Bulletin of Earthquake Engineering, 17: 5583-5607. https://doi.org/10.1007/s10518-018-0370-4

(9) EMS-98. Escala Macrosísmica Europea. Comisión Sismológica Europea (CES). Reykiavik.

(10) Gutenberg, B. y Richter, C.F. (1942). Earthquake magnitude, intensity, energy, and acceleration. Bulletin of the Seismological Society of America, 32(3): 163-191.

(11) Torroja Miret, E. (2007). Razón y Ser de los Tipos Estructurales. Ed. Colegio de Ingenieros de Caminos, Canales y Puertos. Primera edición CSIC, 1957: 189-190, 13, 295, 81.

(12) Miguel Rodríguez, José-Luis de (2019). Estructuras Dos. Madrid: Ed. Munilla-Lería.

(13) Vera Morales, Juan-Antonio (2017). Análisis de los mecanismos de daños en las iglesias de Lorca debidos al terremoto de 2011. En Salcedo, J.C. (Dir.) Cáceres-Florencia, patrimonio vivo: Ensayos técnico-arquitectónicos. Ed. Grupo de Investigación de Construcciones Arquitectónicas (pp. 129-142). Cáreres: Universidad de Extremadura.

(14) Lopes, Mário S., et al. (2018). Earthquakes in central Italy in 2016: comparison between Norcia and Amatrice. En 16th European Conference on Earthquake Engineering (16ECEE).

(15) Sorrentino, Luigi, et al. (2019). Seismic Demand on Historical Constructions During the 2016-2017 Central Italy Earthquake Sequence. In: Aguilar R., Torrealva D., Moreira S., Pando M.A., Ramos L.F. (eds) Structural Analysis of Historical Constructions. RILEM Bookseries, vol 18: 1355-1363. Springer, Cham. https://doi.org/10.1007/978-3-319-994413_145

(16) Lagomarsino, S. and Cattari, S. (2015). Guidelines for seismic performance-based assessment of cultural heritage masonry structures. Bulletin of Earthquake Engineering, 13: 13-47. https://doi.org/10.1007/s10518-014-9674-1

(17) Código Técnico de la Edificación (2006). Real Decreto 314/2006, de 17 de marzo, por el que se aprueba el Código Técnico de la Edificación. Ministerio de Vivienda. BOE $n^{0}$ 74, del 28 de marzo de 2006.

(18) ICOMOS (2005). Recommendations for the analysis, conservation and structural restoration of architectural heritage. International Scientific Committee for Analysis and Restoration of Structures and Architectural Heritage (ISCARS$\mathrm{AH})$, documento aprobado en Barcelona el 15 de mayo de 2005.

(19) Ruggieri, Nicola (2015). L'Ingegneria antisismica nel regno di Napoli (1734-1799). Ariccia, Italia: Aracne Editrice. ISBN: 978-88-548-8564-6.

(20) Vasconcelos, G. y Lourenço, P.B. (2009a). Experimental characterization of stone masonry in shear and compression. Construction and Building Materials, 23(11): 3337-3345. https://doi.org/10.1016/j.conbuildmat.2009.06.045

(21) Durán Ruiz, L.A. (2017). Mejoras estructurales obtenidas en morteros y hormigones de base cal hidráulica mediante la aportación de corcho y fibras de polipropileno para su aplicación en el refuerzo de estructuras históricas. En Salcedo, J.C. (Dir.) Cáceres-Florencia, patrimonio vivo: Ensayos técnico-arquitectónicos. Ed. Grupo de Investigación de Construcciones Arquitectónicas (pp. 143-156). Cáreres: Universidad de Extremadura.

(22) Vasconcelos, G. y Lourenço, P.B. (2009b). In-Plane Experimental Behavior of Stone Masonry Walls under Cyclic Loading. Journal of Structural Engineering, 135(10). https://doi.org/10.1061/(ASCE)ST.1943-541X.0000053 